livraisons

d'Histoire

de l'Architecture

\section{Livraisons de l'histoire de l'architecture}

28 | 2014

Jean-Louis Pascal

\title{
Le cabinet des médailles ou la restitution d'un passé révolu
}

The Cabinet des médailles or the vanished past restitution

Das Münzkabinett oder die Wiederherstellung einer bewegten Vergangenheit

\section{Anne Richard-Bazire}

\section{OpenEdition}

Journals

Édition électronique

URL : http://journals.openedition.org/lha/396

DOI : $10.4000 /$ lha.396

ISSN : 1960-5994

Éditeur

Association Livraisons d'histoire de l'architecture - LHA

Édition imprimée

Date de publication : 10 décembre 2014

Pagination : 159-183

ISSN : 1627-4970

Référence électronique

Anne Richard-Bazire, "Le cabinet des médailles ou la restitution d'un passé révolu », Livraisons de I'histoire de l'architecture [En ligne], 28 | 2014, mis en ligne le 10 décembre 2016, consulté le 19 avril 2019. URL : http://journals.openedition.org//ha/396 ; DOI : 10.4000/lha.396

Ce document a été généré automatiquement le 19 avril 2019

Tous droits réservés à l'Association LHA 


\title{
Le cabinet des médailles ou la restitution d'un passé révolu
}

\author{
The Cabinet des médailles or the vanished past restitution \\ Das Münzkabinett oder die Wiederherstellung einer bewegten Vergangenheit
}

\author{
Anne Richard-Bazire
}

1 De 1865, date de destruction de l'arcade Colbert et de l'ancien cabinet des médailles, à 1917, date de fin d'installation du nouveau cabinet des médailles dans les ailes est et sud du "carré Vivienne», le cabinet des médailles trouve refuge dans les bâtiments construits par Henri Labrouste le long de la rue de Richelieu, de la rotonde appelée jusqu'alors «Voltaire » jusqu'au droit de l'hémicycle de la grande salle de travail. JeanLouis Pascal adapte la rotonde désormais intitulée "Salle des Donateurs", à sa nouvelle destination ; les peintures du mur étaient vouées à la disparition. Pour les préserver, il les recouvre d'un papier légèrement collé, puis d'une toile destinée à recevoir une décoration nouvelle. Encore une fois, Léon Labrouste loua Pascal « pour la délicatesse avec laquelle il traitait en toute occasion les parties achevées de l'édifice »'. À l'extérieur des bâtiments, l'architecte annonce leur destination par un «motif architectural»: des médailles sculptées à la clef des baies donnant sur la rue Vivienne et le jardin. Et l'architecte, poursuivant son œuvre de "bénédictin $»^{2}$, restitue pratiquement à l'identique l'ancien cabinet du Roi installé entre 1741 et 1865 à l'angle nord-est de la Bibliothèque, qu'Henri Labrouste avait détruit.

\section{Le bâtiment sur la rue Vivienne}

2 Pascal reprend sur la rue Vivienne la même ordonnance que sur la deuxième partie de l'aile Colbert: alternance de baies simples et riches à l'étage surmontant un rez-dechaussée à refends (ill. 1). 


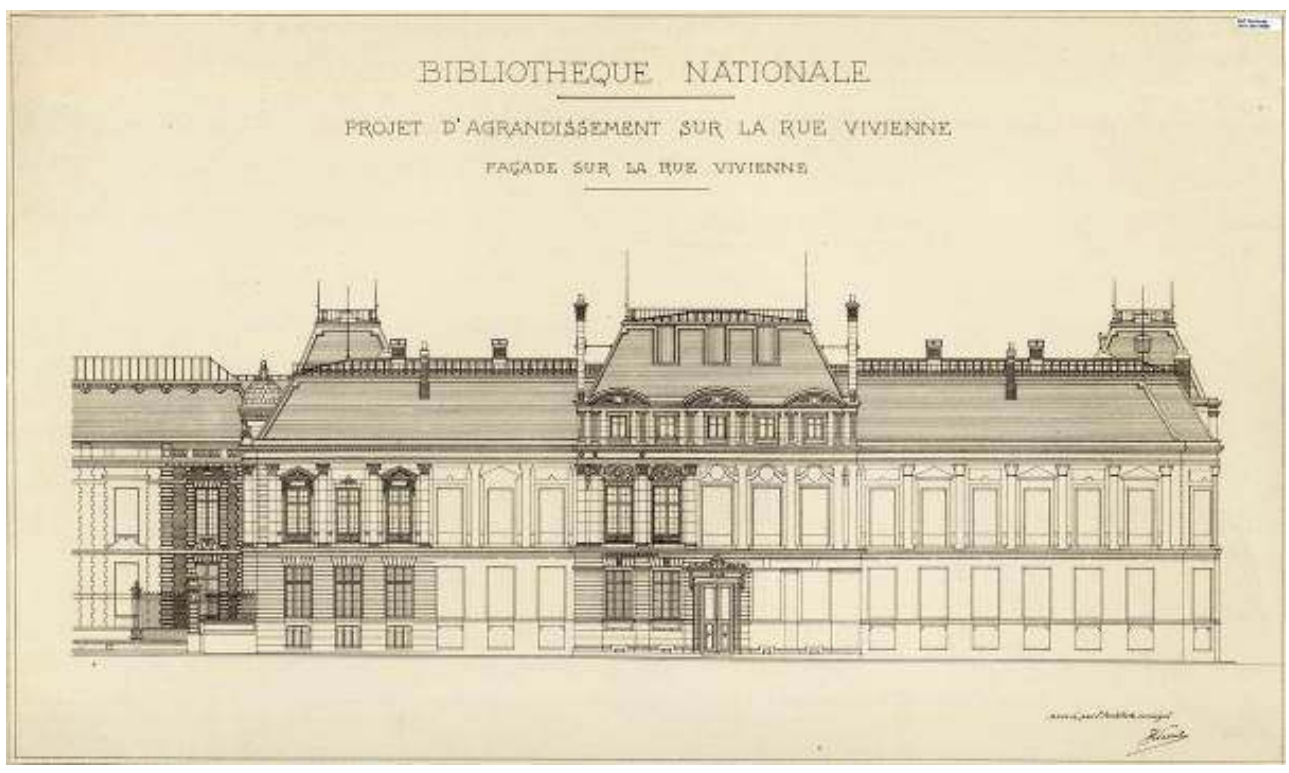

Jean-Louis Pascal. Élévation. Dessin monochrome sur papier. 590 × 992 mm

Arch. adm. B.n.F., 2011/001/4084

3 Sur la façade de la rue Vivienne, dont il tient à ce que les travaux de sculpture soient confiés à Gustave Germain ${ }^{3}$ ( plus exactement la préparation et l'exécution des morceaux du pavillon central sur la rue Vivienne) il emploie de la pierre de Pargny au rez-dechaussée et de la pierre de Villiers-Adam au $1^{\text {er }}$ étage ; « le reste, c'est-à-dire les modèles du rez-de-chaussée et l'exécution des motifs des trois fenêtres de la partie à gauche pouvant être attribués à tout sculpteur et notamment à M. Louis Lavy ${ }^{4}$.

Le motif central est constitué au premier étage de la rue Vivienne par de grandes baies rectangulaires séparées par des colonnes ioniennes engagées et cannelées. Rappelant la vocation de l'édifice, des médailles ont été reproduites à la clef des baies du salon central ${ }^{5}$, enrichies d'une guirlande florale. Elles symbolisent l'art aux grandes époques : Denys de Syracuse pour l'art grec (ill. 2), Galba pour l'art romain, Lionel d'Este pour la Renaissance italienne, Louis XIV pour l'art français ${ }^{6}$ (ill. 2) et une figure de la République française, représentant le XIX ${ }^{\mathrm{e}}$ siècle c'est-à-dire l'époque où l'art de la médaille est redevenu un grand art $^{7}$. 


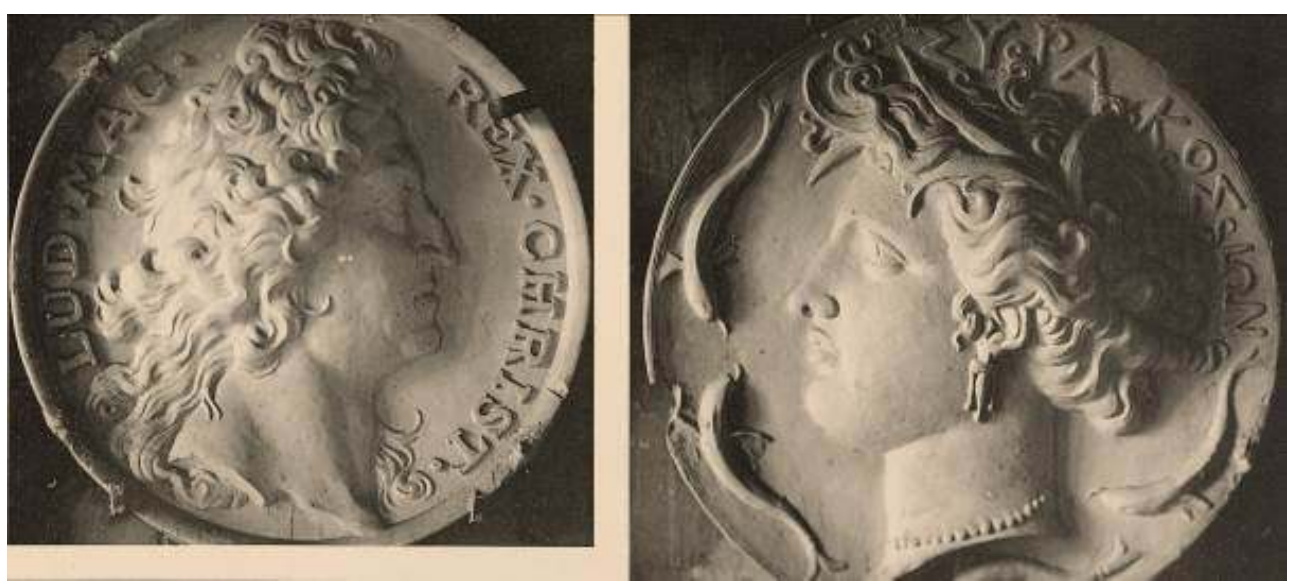

Moulages des médailles de la façade du cabinet des médailles de la Bibliothèque nationale sur la rue Vivienne. Sculpteur Louis Bottée. 1906

Arch. adm. B.n.F. 2007/061/001, pl. 53-54.

Les médailles prévues en bronze seront exécutées en pierre par le sculpteur Louis Alexandre Bottée (1852-1941) :

«J'avais toujours eu l'intention de rendre hommage à la numismatique en accrochant ces beaux spécimens à la clef des grandes baies du salon central, et mon devis général porte une somme de douze mille francs pour des médailles en bronze. L'étude m'a fait décider de les exécuter dans la matière même dans laquelle sont taillées les sculptures et les chapiteaux qui les accompagnent. La couleur du bronze ou la dorure exagèreraient la variété et la richesse relative que comporte cette façade de la Bibliothèque [...] Notre choix n'est pas absolument fait. Au cabinet des médailles, on a mis à ma disposition divers beaux spécimens [...] J'indiquerai les représentations d'art grec, romain, italien de la Renaissance, français, entre lesquelles nous hésitons: Syracuse, Athènes ou Minerve? Un beau type romain: César, Auguste, Néron? Un Malatesta, un Médicis s'il y en avait, un pape pour l'Italie? Pour la France, un Henri IV, un Louis XIV ? Je confierais bien à M. Bottée une tête de la République pour caractériser l'art moderne : il y en a une esquisse intéressante, coiffée d'une tête de coq ; mais je craindrais la comparaison avec tous les chefs d'œuvre énoncés ci-dessus pour lesquels on a le recul du temps »'.

6 Le motif principal d'un bâtiment est destiné à en faire saisir la fonction, dès l'abord ; c'est «l'architecture parlante», telle que Léon Vaudoyer, le premier, l'a définie ${ }^{10}$ que Pascal met en œuvre ici avec des médailles, comme il l'avait fait à la faculté de médecine de Bordeaux en insérant des bustes d'hommes de science entre les colonnes du pavillon central :

«En même temps que je cherchais avec M. Babelon ${ }^{11}$, avec M. Bottée ayant remplacé Chaplain (se déclarant dans l'impossibilité de s'occuper de mon affaire avant un an), j'ai essayé de caractériser les grandes époques de l'art du graveur en médailles par des villes, nobles entre toutes comme Athènes, Rome, Florence, Venise, Paris ; mais nous n'avions pu trouver des types conservés et intelligibles par le passant [...] Votre proposition, M. le sous-secrétaire d'État, est donc en parfait accord avec la pensée directrice de ma composition : honorer les grandes écoles d'art y compris l'art moderne $»^{12}$.

7 Les médailles sont sculptées entre avril 1906 et février $1907^{13}$, avec une interruption entre mai et octobre car l'exposition des "Raretés et pièces exceptionnelles du XVIII ${ }^{e}$ siècle français » qui se déroule dans le cabinet du Roi, oblige à enlever les échafaudages qui en 
obstruent les fenêtres ${ }^{14}$. La maison de serrurerie Baudet et Donon exécute la porte d'entrée de la rue Vivienne ${ }^{15}$ (ill. 3) en fer et bronze, comme elle l'avait fait en 1888 pour l'entrée place Louvois ${ }^{16}$.

\section{3 : Entrée spéciale de la salle Ovale sur la rue Vivienne}

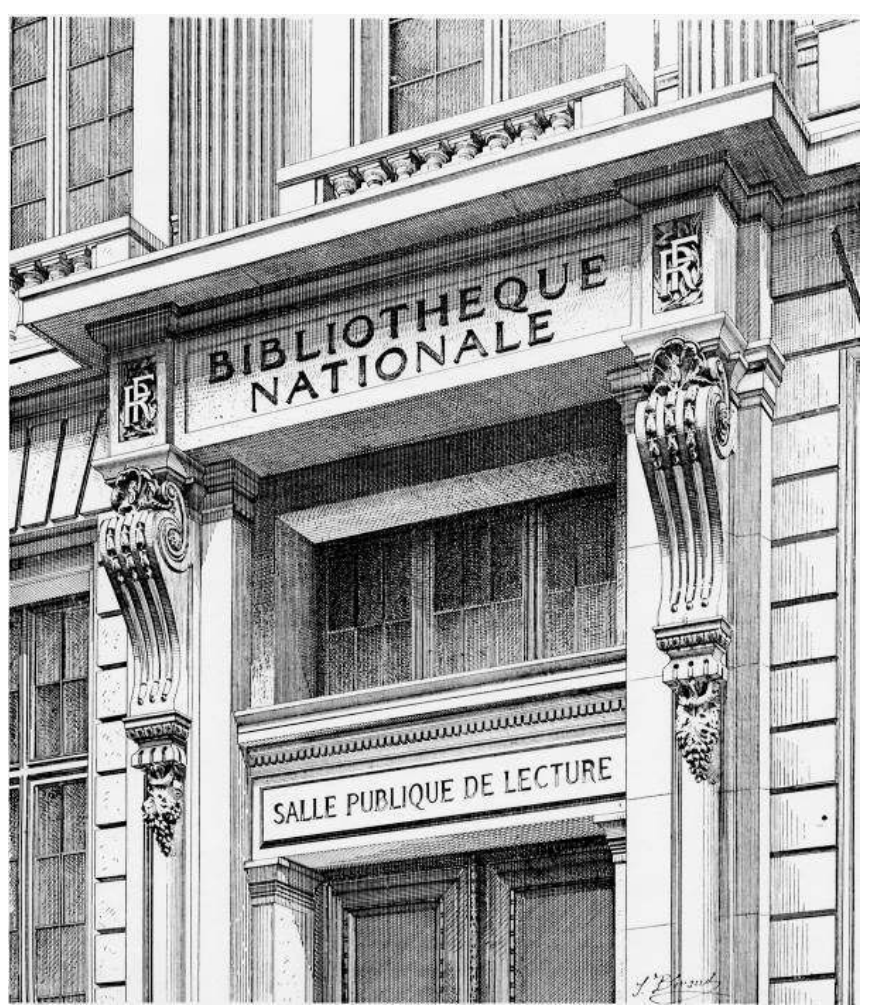

Bibliothèque nationale. Architecte Jean-Louis Pascal. Sculpture décorative : Gustave Germain L'Architecture, 1906, p. 246.

8 La gravure à effet sur pierre de: "Salle publique de lecture » a été exécutée par T. Parizeaux ${ }^{17}$ sur le linteau situé au-dessus de la porte, soit 22 lettres de 0,14 m. La maison Lelièvre Vincent, spécialiste en enseignes, réalise en bronze l'inscription : «Bibliothèque Nationale» au-dessus de l'imposte de la porte d'entrée du vestibule Vivienne ${ }^{18}$. L'entrepreneur de maçonnerie Alexandre Auget propose à Pascal pour les linteaux de la baie de la porte d'entrée : le Comblanchien, le liais de Larrys du Bief, la roche dure de Tercé ou la pierre de Pargny. C'est la pierre dure de Tercé qui est choisie et fournie par l'entreprise Fèvre et $\mathrm{C}^{\mathrm{ie} 19}$. Pascal choisit un granit gris bleu à tons doux pour les colonnes et pilastres destinés au vestibule de la Bibliothèque, rue Vivienne (ill. 4) : «Ces fournitures coûteront en granit certainement moins cher qu'en marbre, avec plus de chances d'être obtenues monolithes dans une gamme de couleur plus variée qu'en cette dernière matière $»^{20}$. 


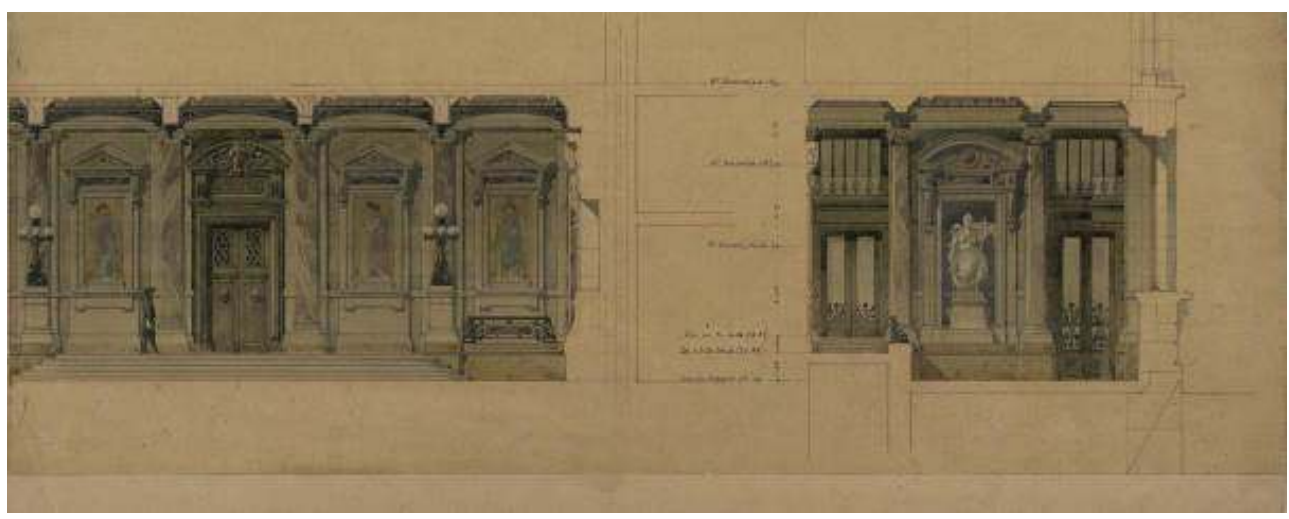

Bâtiment Vivienne, pavillon milieu, rez-de-chaussée. Projet de décoration. Jean-Louis Pascal. Dessin aquarellé sur calque collé sur papier. $299 \times 702$ mm

Arch. adm. B.n.F., 2011/001/4033.

9 À la fin de l'année 1908, Pascal est très ému d'un projet de migration du cabinet des médailles au Louvre: on a logé des manuscrits provisoirement dans deux salles ${ }^{21} \mathrm{du}$ nouveau département des médailles sur la rue Vivienne : le bureau du conservateur et la petite bibliothèque spéciale. Ernest Babelon, conservateur du cabinet des médailles, en est furieux. En effet, cela a entraîné l'installation de fermetures à crémaillères aux fenêtres, que l'on actionne grâce à des perches de trois à quatre mètres de long, empêchant l'installation de bureaux ou de vitrines devant les fenêtres, obligatoire au département des médailles où on travaille à la loupe et à la lumière du jour. Pascal doit convaincre Babelon :

«Pour le cabinet des médailles, afin de pouvoir le reproduire avec son mobilier, avec sa proportion, avec son nombre de fenêtres, avec sa longueur, sa largeur et sa hauteur, j'ai combiné mon plan pour constituer la salle même du cabinet royal. J'ai relevé tout le plancher sur la rue Vivienne pour lui donner la même hauteur, sans tenir compte du résultat de ce changement de niveau dans le comble. J'ai composé ma façade au $1^{\text {er }}$ étage pour signaler aux passants l'existence du cabinet et vous m'avez aidé à choisir les médailles typiques qui figurent au-dessus des fenêtres ; je me propose quelque chose d'analogue pour le retour sur le jardin. Je vous ai fourni des meubles qui doivent se coordonner avec les études faites pour le mobilier d'une autre salle et, en continuant mes études d'arrangement intérieur, je prépare au plafond la glorification justifiée de quelques beaux graveurs modernes en attendant l'hommage rendu aux conservateurs $»^{22}$.

\section{Le bâtiment sur le jardin (ill. 5)}




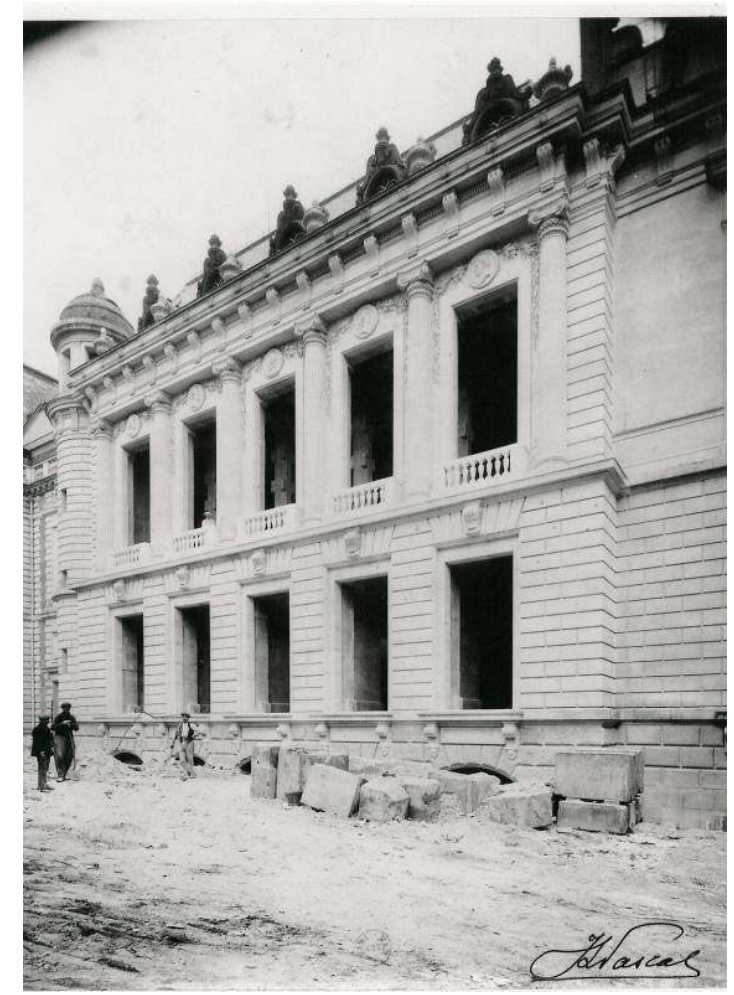

Aile en construction sur le jardin Vivienne. Jean-Louis Pascal. 1912

B.n.F., département des estampes, Ha 132, cl. n²000 B 161849

En 1906, Pascal détruit le corps de bâtiment limitant le jardin au nord, ancienne galerie du Trésor ${ }^{23}$ qui abritait le service des télégraphes et une collection ethnographique de la Bibliothèque nationale en 1878. Construit en pierre, il comprenait un rez-de-chaussée et un $1^{\mathrm{er}}$ étage couvert en ardoise et zinc ${ }^{24}$. Il prévoit pour 1907 l'achèvement des façades est et midi du pavillon sur le jardin ${ }^{25}$ et pense que le plancher du rez-de-chaussée du bâtiment sur le jardin ne sera pas achevé avant la fin du $1^{\mathrm{er}}$ semestre $1909^{26}$. À l'été 1910 , le mur du bâtiment sur le jardin a atteint le bandeau du rez-de-chaussée ${ }^{27}$. À la fin de l'année 1911, on termine les ravalements extérieurs des bâtiments sur le jardin ${ }^{28}$. Germain a réalisé toute la sculpture décorative ; il a fourni le modèle des cinq lucarnes en zinc, réalisées par L. Bonfils, qui ornent la toiture sur le jardin. Ce sont de grandes lucarnes ornées de volutes de feuilles d'acanthe et de chutes de feuillage de chêne et de rubans; elles sont sommées d'une pomme de pin sortant d'un cornet à canaux ${ }^{29}$. Dans le vestibule sur le jardin, Pascal utilise de la pierre de Villiers-Adam jusqu'à l'arase de pose du plancher haut du premier étage ${ }^{30} \mathrm{y}$ compris pour la tourelle ${ }^{31}$, dont la coupole à écailles due à Albert-Raymond Germain ${ }^{32}$ prévue en pierre de Tercé, est en pierre de Lavoux à grains ${ }^{33}$. Surmontant l'entablement et le chéneau orné de palmettes et situés en avant de la toiture du bâtiment sur le jardin de la Bibliothèque, quatre vases en pierre de Tercé avec anses à palmette s'intercalent entre les lucarnes.

11 Pour la façade sur le jardin, dont le motif décoratif est semblable à celui de la façade sur la rue Vivienne, les médailles, accompagnées d'un double motif de guirlandes en pierre de Villiers Adam entourant la partie haute des fenêtres, représentent les profils de François $1^{\mathrm{er}}$, Henri IV, le cardinal de Richelieu, Louis XV, Napoléon $1^{\mathrm{er}}$. Ils symbolisent les époques 
riches pour l'histoire de la Bibliothèque ${ }^{34}$. Jean-Louis Pascal y « donne la main à François Mansart » en accordant la façade moderne et le style Louis XIII de la galerie des estampes. C'est le style « néo-dix-septième » administratif que l'on voit fleurir à partir de 1860 sur les édifices publics. Pour réussir ce raccord, Pascal doit choisir pour la dernière travée du bâtiment après la tourelle, une brique qui ne dépare pas la brique ancienne de la galerie Mazarine ; l'inspecteur préféré de Pascal à la Bibliothèque, Louis Marc Emery ${ }^{35}$, demande à Alexandre Auget, l'entrepreneur de maçonnerie, son avis: «Pour l'échantillon de brique pouvant se raccorder avec celle apparente de la galerie Mazarine, elle est toute trouvée : c'est de la brique tête noire moule d'acier, il n'y a que les maisons Baudelot et Fouinat qui en ont. Pour la brique Vaugirard ${ }^{36}$, elle est [entièrement] de $1^{\text {er }}$ choix, on ne connaît pas de $2^{\mathrm{e}}$ choix chez les fournisseurs. Je suis bien d'accord avec vous pour vous fournir de la Belleville, mais je tiens à vous prévenir qu'on a de la brique [d'] un peu toutes les dimensions avec plus ou moins de chaux $»^{37}$.

\section{L'escalier d'honneur}

En 1903, Pascal commence à se préoccuper de la construction du grand escalier (ill. 6) qui permettra d'accéder au nouveau département des monnaies et médailles :

«Dans les problèmes variés qui se présentent pour les raccordements des constructions nouvelles avec les parties plus ou moins anciennes de la Bibliothèque, je trouve comme amorce du futur escalier avec lequel j'aurai à me raccorder et dont je rencontre déjà un morceau, de l'Euville, de l'Hameret et du Charentenay. C'est le Charentenay qui paraît devoir donner le moins mauvais raccord et je me souviens que $\mathrm{M}$. Vernaud, qui fut mon entrepreneur à la Bibliothèque en même temps qu'il travaillait à l'Hôtel de Ville préconisait fort cette pierre et son Courson, non moins blanc. L'emploi que j'en veux faire est le rez-de-chaussée : c'est dire que je voudrais que sa résistance fût équivalente au Pargny que j’ai en général au rez-de-chaussée » ${ }^{38}$. 
III. 6 : Escalier d'honneur de la Bibliothèque nationale

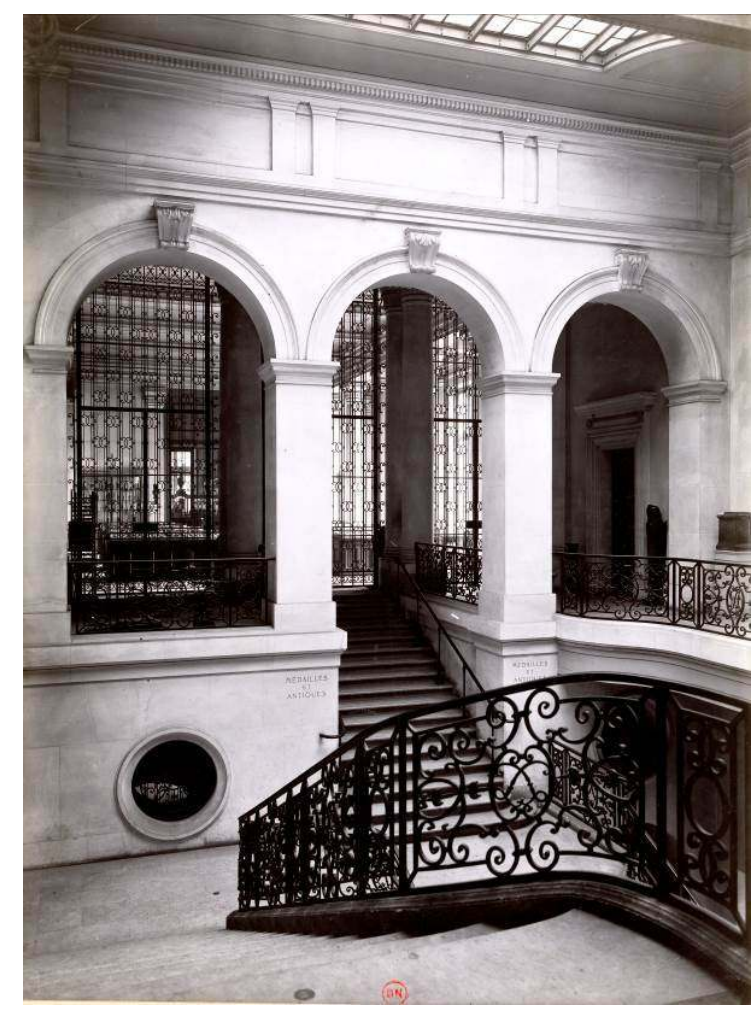

Jean-Louis Pascal et Ernest Recoura. La volée tourne à gauche ; Serge Macel, architecte de la Bibliothèque depuis 1981 l'inversera vers la droite en 1987

B.n.F., département des estampes, Va 237 (7) Fol H 23519-0016.

En attendant, l'architecte est obligé de demander en décembre $1903^{39}$ à son menuisier Favarou de lui fournir un escalier provisoire en bois pour accéder à la salle du département des médailles (le cabinet du $\mathrm{Roi}^{40}$ ) où se déroule en 1904 l'exposition des Primitifs français. En 1905, il indique à son vérificateur Didelot: « Nous avons dans les murs de l'attique du motif central sur la rue Vivienne un mélange de Villiers-Adam et de Banc royal de Méry de même que sur les assises posées de la partie de la salle ovale, voisine du vieux bâtiment des manuscrits et de l'escalier ancien avec lequel nous nous raccordons".

En $1906^{41}$, Pascal réitère sa demande à Favarou de location d'un escalier provisoire et d'une charpente pour accéder à une nouvelle exposition sur les œuvres d'art du XVIII siècle qui se tient toujours dans le cabinet du Roi, seule pièce du futur cabinet des médailles dont Pascal a alors achevé la restitution. Il n'y a toujours pas d'escalier menant au nouveau département des monnaies et médailles.

Le 30 avril 1908, Pascal commence à démolir l'ancien escalier du bâtiment du fond de la cour d'honneur ${ }^{42}$. Le 23 mai de la même année, il demande au sous-secrétaire d'État aux beaux-arts, Dujardin-Beaumetz, de le débarrasser au plus vite des gravats occasionnés par cette opération : " La démolition des parties du vieil escalier à remplacer par le nouveau destiné à relier les parties anciennes de l'établissement avec les nouvelles a accumulé des gravois [...] Les moellons durs ou tendres sortis des parties de révolutions de l'ancien emmarchement constituent un amoncellement [...] et je demande à $\mathrm{M}$. le sous-secrétaire 
d'État de bien vouloir en aviser le Domaine d'en faire la vente le plus vite possible $»^{43}$. Trois jours plus tard, Dujardin-Beaumetz répond à Pascal: «Je viens de prier $\mathrm{M}$. le ministre des finances de donner des instructions en vue de la mise en adjudication de matériaux inutilisables provenant de la démolition du vieil escalier à la Bibliothèque nationale $»^{44}$.

S'étant adressé à son maçon habituel, Alexandre Auget, Pascal se voit refuser la fourniture de pierre de Charentenay : «[J’ai la] surprise désagréable [...de votre annonce] de la difficulté de vous procurer du Charentenay pour le complément de l'escalier commencé il y a un demi-siècle avec cette pierre ${ }^{47}$. L'architecte s'en plaint à DujardinBeaumetz : «Le premier [Auget] me déclarait qu'il lui était impossible de se procurer de la pierre de Charentenay pour le raccordement avec les parties des murs de l'escalier amorcé par mon prédécesseur Labrouste, et que mon enquête immédiate démontrait que cette affirmation n'avait aucune base $\aleph^{48}$. Pascal a donc détruit le vieil escalier qui permettait d'accéder au département des manuscrits et il s'est raccordé à l'amorce de l'escalier réalisée par Henri Labrouste. Le grand escalier de la Bibliothèque nationale est donc l'œuvre de trois architectes : Henri Labrouste, Jean-Louis Pascal et Ernest Recoura, qui prend la suite de Pascal en mars 1912. Nous trouvons en effet dans le récit de Léon Labrouste sur la construction de la Bibliothèque :

«Les travaux du pavillon destiné au vestibule des estampes marchaient vers leur fin en 1870 et l'amorce du grand escalier était commencée. Cet escalier devait occuper le même emplacement que l'ancien datant de l'époque de Mazarin. [...] L'année 1871 vit [...] les amorces du grand escalier avec ses deux gaines à ceinture de bronze, surmontées chacune d'une tête de génie; l'arrivée de l'escalier au premier étage ${ }^{49}$, les balustrades en pierre à draperie et l'ajour du plancher de ce même étage ${ }^{50}$ avec son garde-corps en fer forgé, les portes d'accès, etc., qui furent terminés tandis que [...] le bâtiment neuf sur le square Louvois, le pavillon d'angle et l'amorce sur la rue Colbert étaient continués. " $^{51}$

En mars, Pascal se plaint de l'incorrection du tracé circulaire du limon rampant du grand escalier $^{52}$. Au début du mois d'août 1911, Ernest Hébrard ${ }^{53}$ informe Pascal que le plafond vitré destiné au dessus de l'escalier et son limon sont livrés ${ }^{54}$. Dans un petit point fait par Ernest Recoura au sous-secrétaire d'État le 29 avril 1912, il indique qu'il reste à terminer le grand escalier ${ }^{55}$, notamment à décorer les parois latérales ${ }^{56}$. Les ravalements ne sont toujours pas réalisés en février 1913 : « [Il reste à faire] dans le vestibule du $1^{\text {er }}$ étage sur le jardin et dans le grand escalier : ravalement en plâtre et sur pierre des plafonds, murs, colonnes, etc $»^{57}$.

19 À l'extrémité du vestibule d'entrée, le grand escalier permet de monter à mi-étage, par une volée droite, au cabinet des médailles et antiques d'une part; il débouche d'autre part au premier étage sur un grand vestibule sur lequel s'ouvrent la salle de travail des manuscrits, la galerie Mazarine, et la terrasse longeant la salle de lecture du département des imprimés et aboutissant à la galerie de la réserve. Pour le dessin de la rampe, Pascal s'inspire de la rampe en fer forgé de l'ancien escalier de la Bibliothèque qui se trouvait dans le corps de logis longeant la rue de Richelieu (ill. 7) ; en effet Henri Labrouste avait détruit ou vendu plusieurs pièces de style Louis XV dont une rampe en fer forgé, orgueil de la Wallace Collection à Londres : «Il en aura été de la grille de la place Royale comme de la magnifique rampe de l'escalier de la Bibliothèque nationale que j'ai vue récemment 
à Londres [raconte Pascal], au nombre des plus beaux motifs de la collection Richard Wallace.

III. 7 : Rampe de Hertford House, résidence de la Wallace collection, Londres

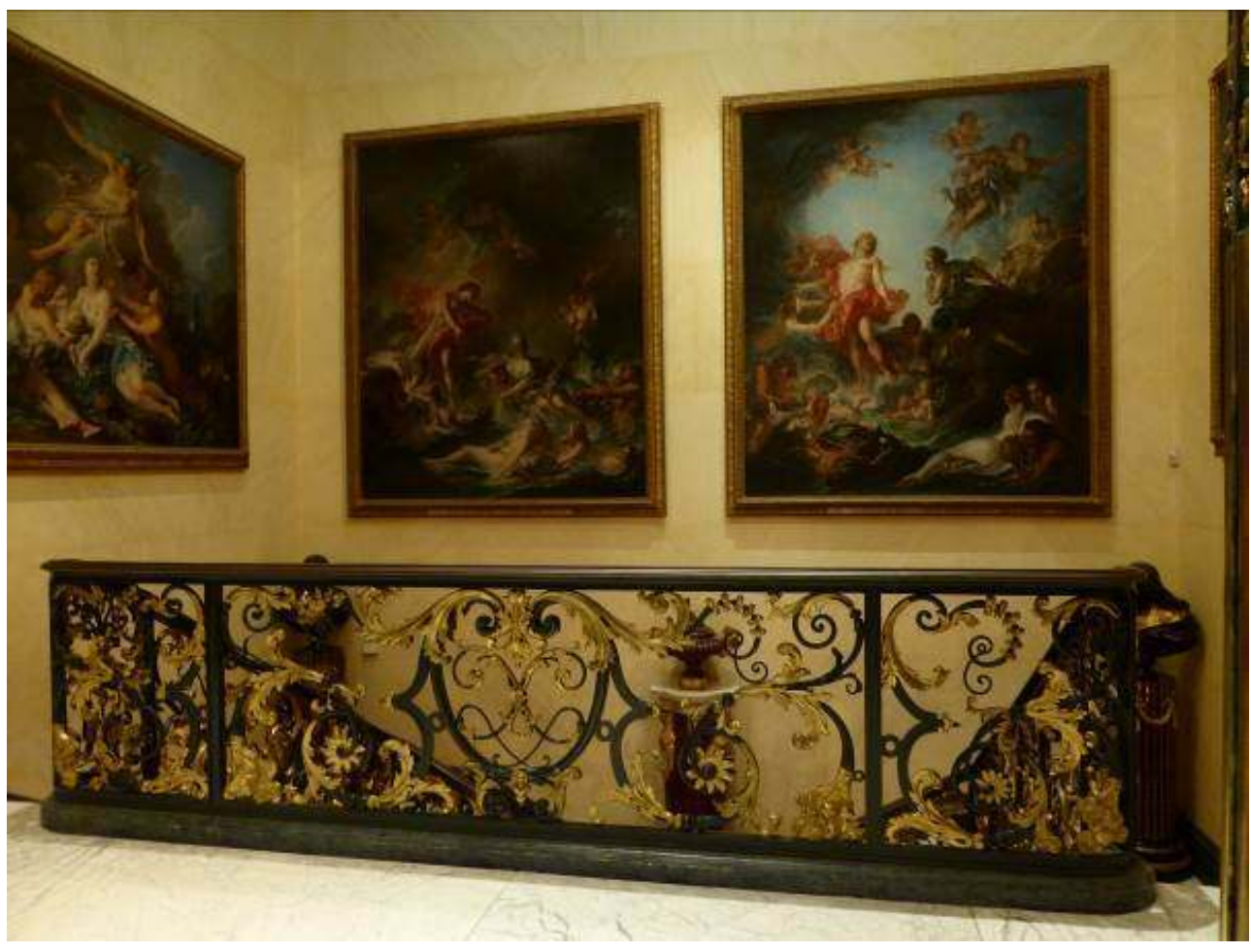

Fer forgé et bronze doré. Rampe de l'escalier de l'ancien palais de Mazarin, enlevée à la bibliothèque entre 1868 et 1874 par Henri Labrouste et vendue à sir Richard Wallace qui la fit adapter par la firme parisienne de Geslin dans son hôtel d'Hertford House en 1874

Cl. auteur. Juillet 2013.

On l'a vendue. Comment en ai-je un seul panneau à mon agence ? Il suffit à donner un profond regret de cette perte qui aurait pu être réparable ${ }^{58}$. La maison Baudet et Donon exécute la porte en fer du cabinet des médailles ; les ornements en bronze en sont fournis par la maison Jaboeuf et Rouard ${ }^{59}$.

\section{Les quatre premières salles du département des médailles}

Dans le projet primitif, la salle qui servait de vestibule d'accès au département des médailles, grande salle du premier étage sur le jardin, avait son plancher, que Pascal prévoyait en ciment armé ${ }^{60}$, percé de deux grands oculi destinés au logement de la partie supérieure des globes de Coronelli installés dans la salle des globes située en-dessous. Elle ne comportait aucun mobilier. On l'appelait « le balcon des globes Coronelli » (ill. 8). 


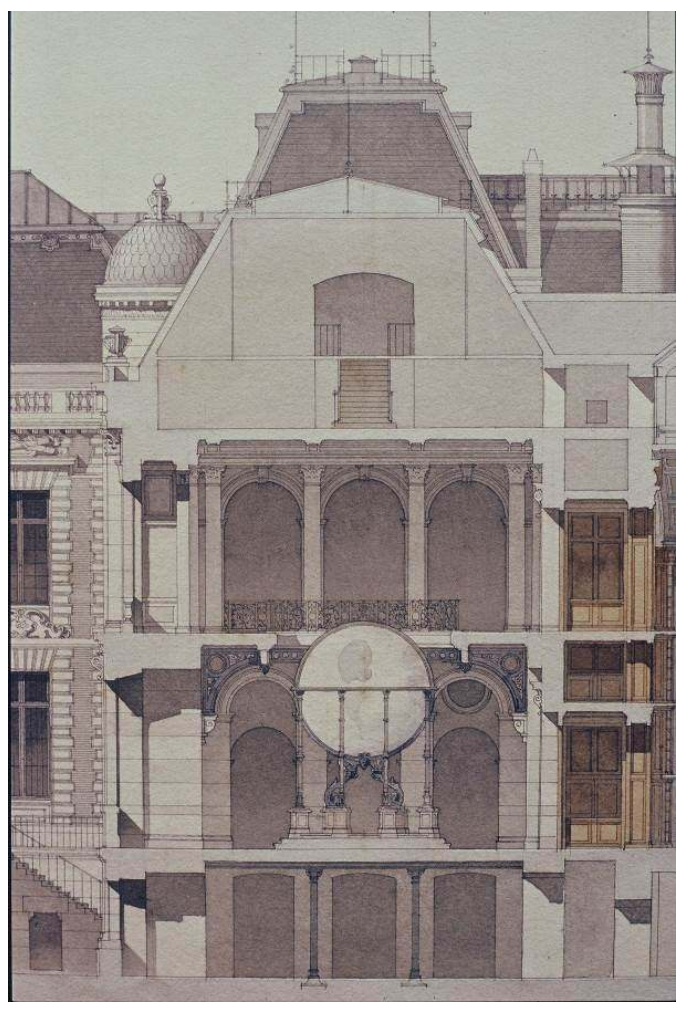

JEAN-LOUIS PASCAL. LAVIS, TEINTES PLATES DE COULEUR ET AQUARELLE

ARCHIVES NATIONALES ; CARTES ET PLANS. VA/195/26. CL. J.-L. CAgNIN

Elle devait être utilisée comme musée épigraphique, ses parois en pierre devant s'orner de fragments antiques scellés dans les murs. En 1914, la suppression du musée épigraphique et des oculi à la suite du transfert des globes à Versailles ${ }^{61}$ laisse libre la vaste pièce du premier étage. Des vitrines sont disposées le long des parois et transversalement, au droit de chaque point d'appui, avec passage central ${ }^{62}$. C'est l'actuelle salle des colonnes ${ }^{63}$ toute blanche et dallée, d'une architecture sobre, éclairée par de hautes fenêtres sur le jardin; elle contient bijoux, médailles, objets égyptiens provenant de collections célèbres. La salle qui lui fait suite, à l'angle du bâtiment, ouvre par une baie sur le jardin et par trois baies sur la rue Vivienne : c'est la salle du Grand Camée ${ }^{64}$. Elle renfermait les plus belles pièces des trésors personnels des rois de France, parmi lesquels le Camée antique de la Sainte-Chapelle et le fauteuil de Dagobert. Cette salle est de couleur grise assez soutenue avec mouluration et décoration en relief rehaussée d'or comme celle qui lui fait suite, la salle de Luynes. Son plafond est orné sur le pourtour de branches d'oliviers et de lauriers et aux angles, quatre gros médaillons circulaires cernés d'oves, blancs et or, dont Pascal a dessiné le modèle dans une lettre à Gustave Germain (ill. 9), figurent des médailles; ils représentent au S.E. : la médaille de la défense de Paris lors du siège de 1870 représentée par une femme coiffée d'une ville crénelée tenant un fusil (ill. 10) ; à ses pieds, un canon; au loin les fortifications entourant Paris ; au N.E. : une semeuse, au N.O. : une médaille « à la mémoire des élèves de l'École des beaux-arts morts pour la défense de la patrie, 1870-1871 »; on y voit une femme voilée tendant la palme du martyr à un jeune homme allongé avec un drapeau ; au S.O. : un Apollon ailé sculpte avec marteau et ciseau la tête d'un dieu barbu, peut-être Jupiter. Pascal demande à Gustave Germain d'en réaliser les esquisses : 
«Il faudrait que nous pussions préciser comment s'exécuteront les médailles dont je fais les motifs des quatre angles. Si nous n'avions cherché qu'un motif à répéter quatre fois: faisceau de licteur, couronnes, etc, la chose s'arrangerait plus facilement qu'avec la variété que j'ai décidée [...] déjà Chaplain et Roty ont fixé leur choix sur les médailles à reproduire. Pour le premier, il ne croit pas pouvoir retrouver le moule de la médaille de la défense de Paris et il m'a prêté une réduction de 0,10 $\mathrm{m}$ de diamètre. [...] Pour Roty, un mot de lui me dit qu'il va faire rechercher par sa femme un plâtre qu'il doit avoir "dans une grandeur qui pourra m'être utile" [...] Pour les deux autres médailles, je vais chercher ce soir ce que j'ai de possible de Chapu et de Degeorge ${ }^{65}$ au cabinet des médailles, [...] et je m'adresserais ailleurs si je ne trouvais pas de sujet pouvant avoir quelqu'analogie d'échelle avec les deux premiers. [...] Des têtes auraient bien pu faire l'affaire, malgré la grandeur de l'échelle? Aussi est-il peut-être nécessaire, d'une façon générale, de tâter par une présentation en place de l'effet plus ou moins préférable qu'on aurait d'une tête ou d'un motif de composition avec un ou plusieurs personnages. Pensez-y et donnez m'en votre avis. " ${ }^{66}$

III. 9 : Dessin du modèle pour les médaillons ornant les quatre coins de la salle du Grand Camée du cabinet des médailles de la Bibliothèque nationale

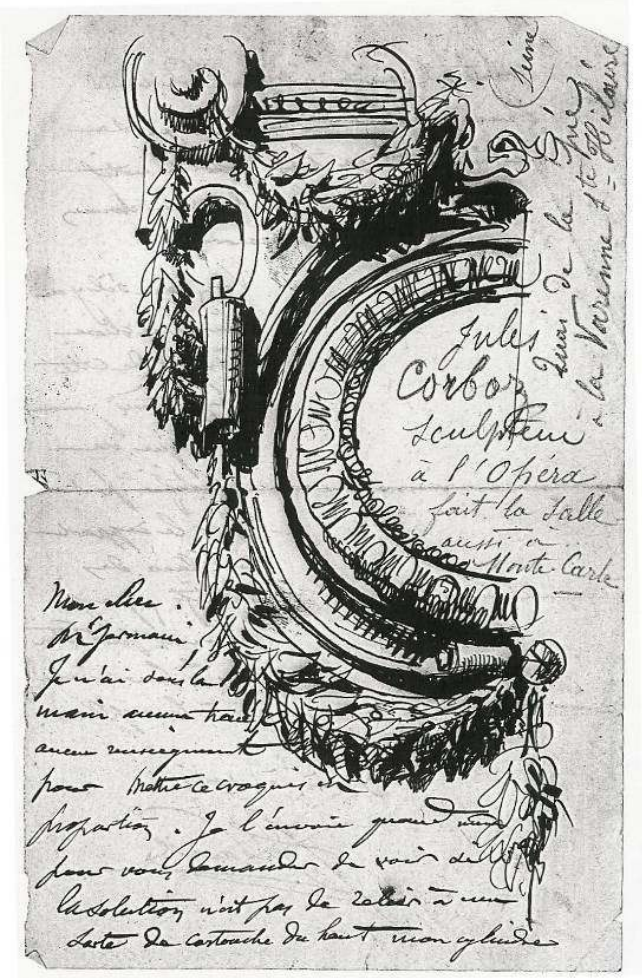

Jean-Louis Pascal. Lettre de Jean-Louis Pascal à Gustave Germain, non datée

Collection Patrice et Alexandra Germain. Cl. auteur. 


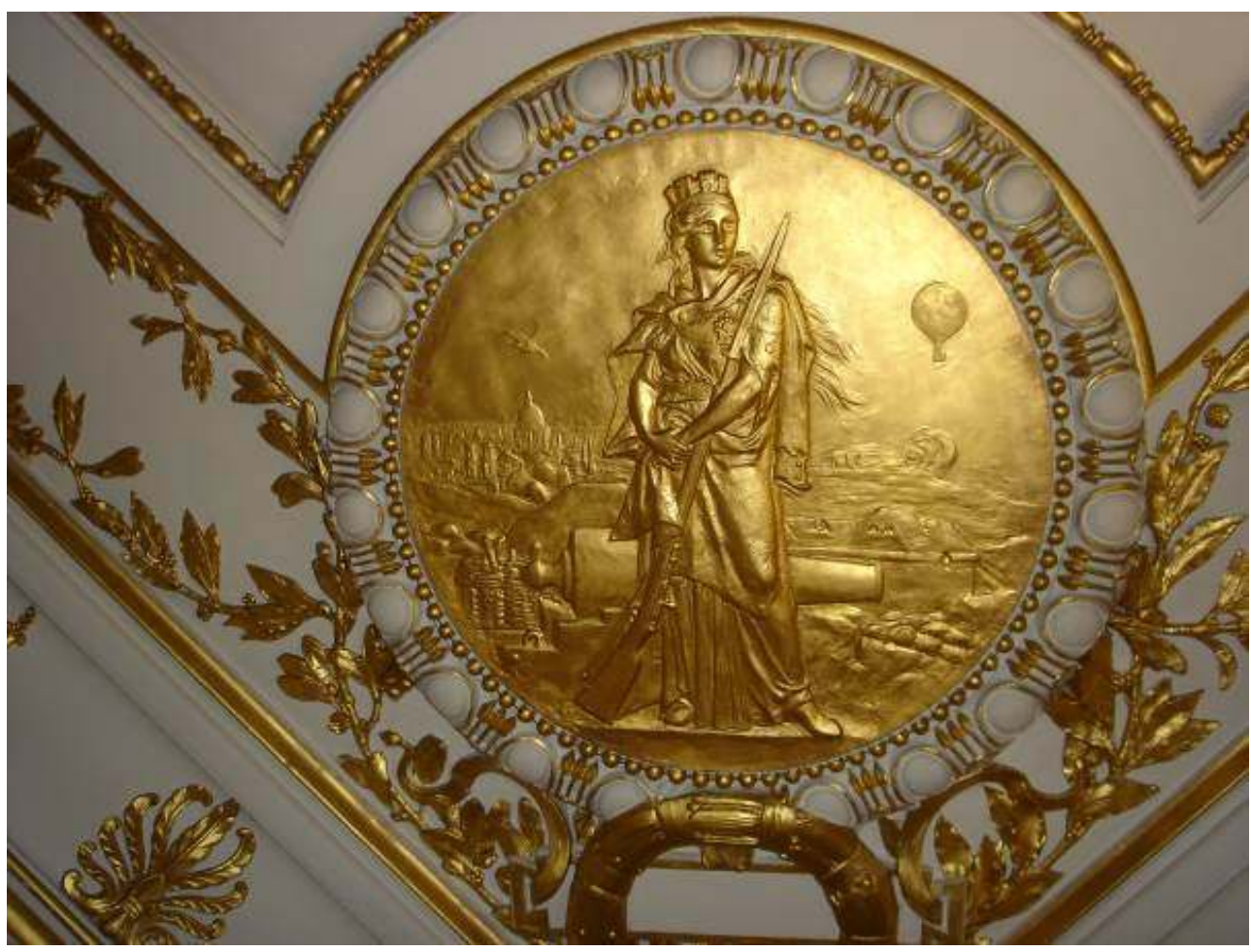

Médaillon décoratif. Salle du Grand Camée du cabinet des médailles. Conception Jean-Louis Pascal $\mathrm{Cl}$. auteur.

La Bibliothèque impériale fut autorisée, par un décret rendu sur proposition du ministre d'État le 30 novembre 1862, à accepter le don que le duc de Luynes lui fit de magnifiques collections comprenant médailles, bijoux, statuettes, objets d'art antiques, vases étrusques, épée de Boabdil, dernier roi maure de Grenade ${ }^{67}$. Celles-ci devaient être logées dans une salle unique, ce fut la salle faisant suite à celle du Grand Camée ${ }^{68}$. Puis vient la salle Barthélémy ${ }^{69}$ éclairée par cinq fenêtres sur la rue Vivienne, et bordée sur trois de ses côtés par deux étages de médailliers surmontés d'un étage de rayonnages de livres avec balcons. Elle est meublée de deux tables avec médailliers en acajou rehaussé de cuivres dorés ${ }^{70}$. Les armoires en acajou, avec incrustations de cuivre, furent réalisées par Tony Selmersheim ${ }^{71}$.

\section{La restitution du cabinet du Roi.}

\section{LE CABINET DU ROI DE L'ARCADE COLBERT (1741-1865)}

Décidé dès la Régence, le retour du cabinet des médailles de Versailles à la Bibliothèque ne fut effectif qu'en 1741, date à laquelle il s'installe dans l'hôtel de la marquise de Lambert. Il avait fallu attendre le décès de celle-ci en 1733 pour annexer ses appartements situés à l'extrémité de l'ancien hôtel de Nevers; on y logea le cabinet des médailles. Au premier étage, l'architecte de Cotte rétablit l'unité originale de la galerie en détruisant les cloisons ; il perça quatre fenêtres sur la rue de Richelieu et unifia celles qui donnaient sur la rue Colbert. Le cabinet des médailles occupait les deux-tiers de l'ancien salon de la marquise, le dernier tiers étant donné à la galerie des imprimés qui lui faisait suite, et 
l'ancienne salle à manger de la marquise, soit tout le premier étage de la galerie au-dessus du mur sud de l'arcade Colbert :

«Le nouveau cabinet des médailles était un rectangle deux fois plus long que large. À l'est et à l'ouest, le mur était percé de quatre fenêtres qui délimitaient trois trumeaux d'égale dimension. Au nord et au sud, deux hautes portes encadraient un important trumeau central. La porte sud-ouest, disposée pour la symétrie, dissimulait sans doute un placard. La porte sud-est ouvrait seule sur la galerie des imprimés, la porte nord-ouest sur le bureau du garde des médailles, la porte nordest sur un vestibule où débouchait un escalier construit par Robert de Cotte. L'accès des visiteurs de marque se faisait par la galerie des imprimés, celui du tout-venant par l'escalier. La pièce était entièrement revêtue d'un lambris sculpté ${ }^{72}$. [...] Les six médailliers appuyés aux trumeaux sont d'un modèle identique. Il s'agit chaque fois d'une console d'applique supportant une table de marbre rose chantourné, sur laquelle repose un placard à deux battants. La console est formée d'une ceinture et de deux pieds reliés par une entretoise dont le décor fouillé contraste avec la sobriété des vantaux des placards. Les deux grands médailliers possèdent une construction de même type : leur console est dotée de six pieds, les quatre pieds médians étant reliés par une entretoise en " $X$ " surmontée d'un brûle-parfum. On compte quatre vantaux au lieu de deux. Complétant le mobilier, une grande table occupe le centre de la pièce; des chaises cannées estampillées Louis Cresson [1706-1761] sont rangées autour d'elle. " ${ }^{73}$

Des tableaux avaient été commandés par Robert de Cotte pour décorer cet espace. De concert avec l'abbé Bignon et le duc d'Antin (1665-1736), directeur général des bâtiments du Roi, il avait décidé de choisir dans la mythologie les sujets convenant le mieux «au temps des sciences et de la littérature » : Apollon et les neuf muses. Les trois trumeaux situés entre les quatre fenêtres donnant sur la rue de Richelieu furent décorés par Carle Van Loo (1705-1765) et présentés au Salon de 1745 (ill. 11). 
III. 11 : La Poésie Amoureuse ou Psyché conduite par l'Hymen, Apollon, Mercure et Hercule Musagète, Euterpe et Pan

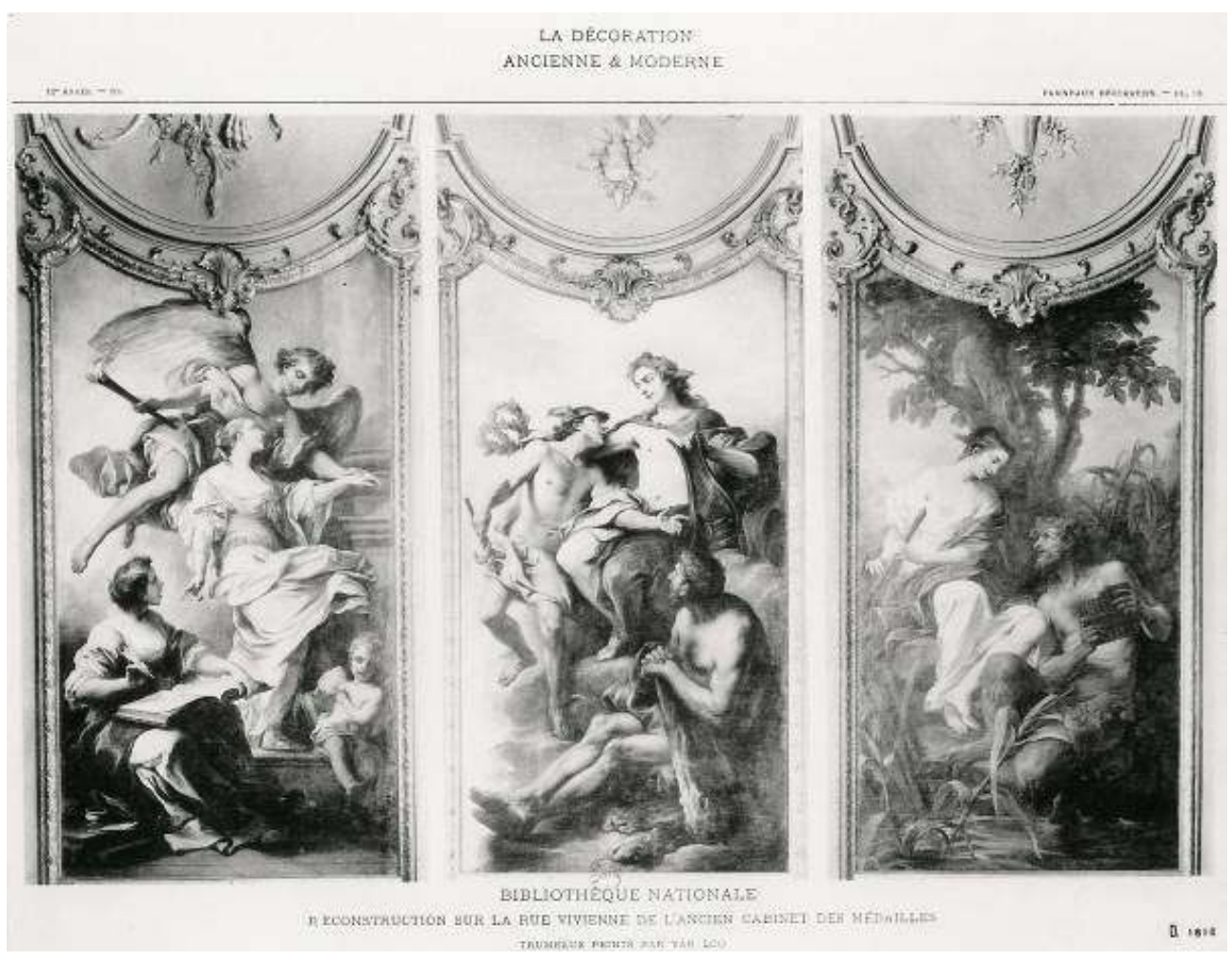

Peintures de Carle Van Loo. 1745. Mur ouest du cabinet du Roi reconstitué par Jean-Louis Pascal au nouveau cabinet des médailles

B.n.F., département des estampes, Ha 132, cl. n²000 C 230716

Le premier trumeau à gauche représentait Polymnie ou la poésie amoureuse : en haut de la toile, l'Hymen ailé armé d'une torche saisit le bras de Psyché pour la mener dans l'Olympe ; en bas, Polymnie consigne les détails de la scène dans un livre, un petit amour assis à ses côtés. Dans le trumeau central, Mercure, sous les traits du duc de Richelieu, rend compte à Apollon, qui a les traits de Louis XV, de l'arrivée de Psyché; Hercule assis jambes croisées regarde les deux autres divinités. Le dernier trumeau représente Euterpe, inventrice de la flûte, avec auprès d'elle « des roseaux agités par les vents, première origine de l'harmonie ». Dans la campagne, au bord d'une rivière, Euterpe tenant sa flûte et Pan avec sa syrinx se regardent avec complaisance. Les trois trumeaux placés du côté de la rue Colbert ont été réalisés par Charles Joseph Natoire et présentés au Salon de 1745 (ill. 12). 


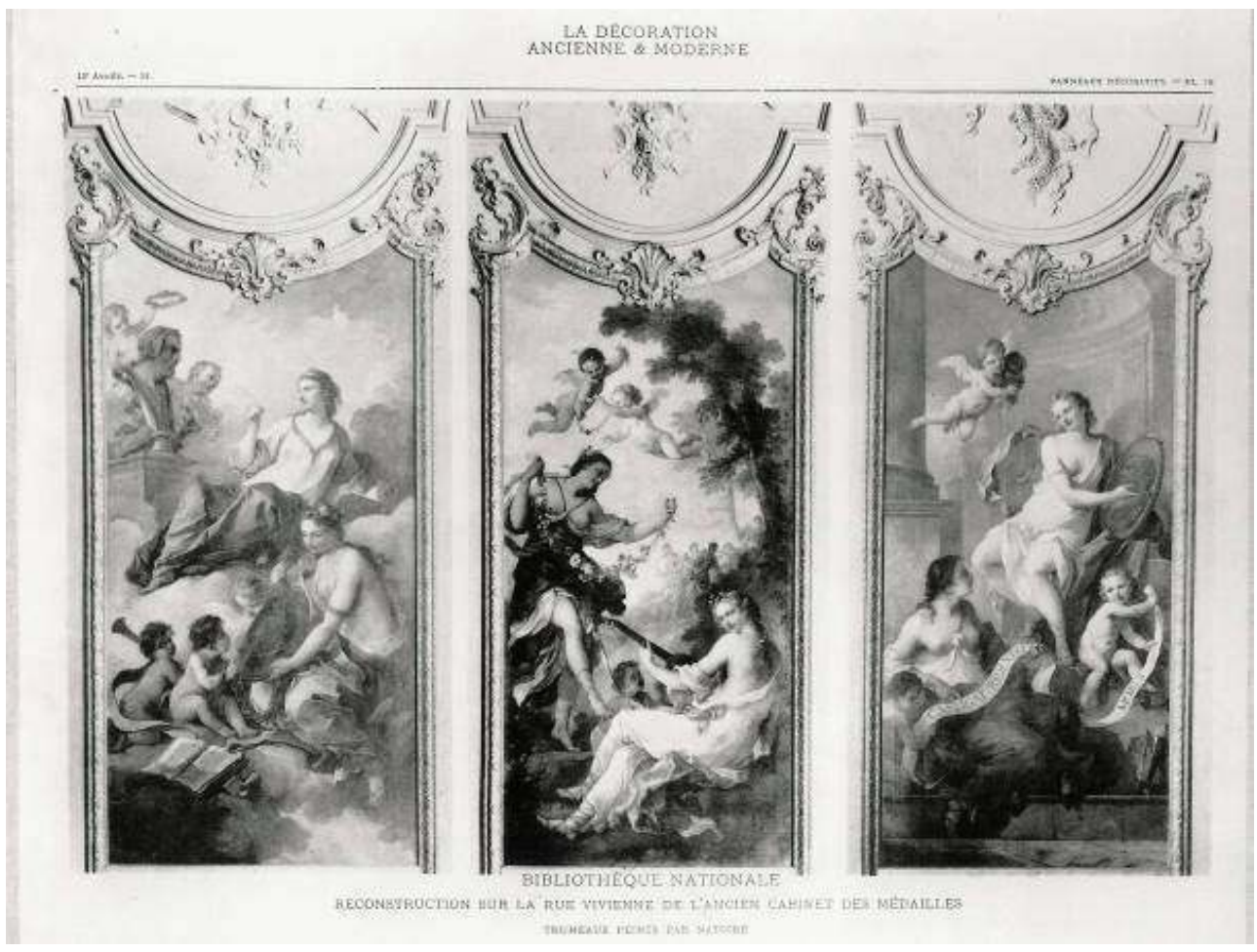

Peintures de Charles Natoire. 1745. Mur est du cabinet du Roi reconstitué par Jean-Louis Pascal au nouveau cabinet des médailles

B.n.F., département des estampes, Ha 132, cl. n²000 C 230716

Dans le premier trumeau, Thalie ou la Comédie est dédoublée, sous les traits d'une jeune fille blonde tenant un médaillon orné du profil de Térence dans le haut du tableau, sous ceux d'une femme brune dans la partie inférieure tenant un phylactère sur lequel est écrit : « Castigat ridendo mores ». Dans le panneau central, Calliope ou la Poésie épique est présentée aussi sous l'aspect de deux jeunes femmes: en haut l'une lève sa plume pour regarder le buste d'Homère autour duquel volettent deux amours, l'autre jeune femme présente le médaillon de Virgile à trois autres amours. Dans le dernier trumeau, Terpsichore ou la Danse, jeune femme brune parée de tresses et de guirlandes dans le haut du tableau, se mue en blonde joueuse de guitare regardant le spectateur, dans le bas.

Les petits côtés de la galerie étaient décorés au sud par le tableau de Louis XIV de Hyacinthe Rigaud, au nord par le Louis XV de Louis Michel Van Loo. Quatre dessus-deporte peints par François Boucher les encadraient. Au sud, Clio et Melpomène, au nord, Uranie et Erato. Clio (présenté au Salon de 1743) écrit l'histoire de Louis sur un grand livre porté par les ailes du Temps, en regardant un profil de Louis XV sur un médaillon de terre cuite tenu par deux amours; un amour numismate observe des médailles à la loupe. Melpomène, le visage levé vers le ciel brandit un poignard où sont enfilées des couronnes et de sa main droite désigne sur un livre les noms de Virgile et d'Homère ; un amour tient une trompette, un autre tresse une couronne de lauriers. Au nord, Uranie recevant ses attributs de trois amours au sommet d'un rocher et Erato, (présenté comme le tableau précédent au Salon de 1746) désignant un recueil de musique avec un caducée, encadrent le tableau de Louis XV. 


\section{La restitution du cabinet du Roi dans le nouveau bâtiment de la rue Vivienne (ill. 13)}

\section{13 : Le cabinet du Roi reconstitué au cabinet des médailles de la rue Vivienne}

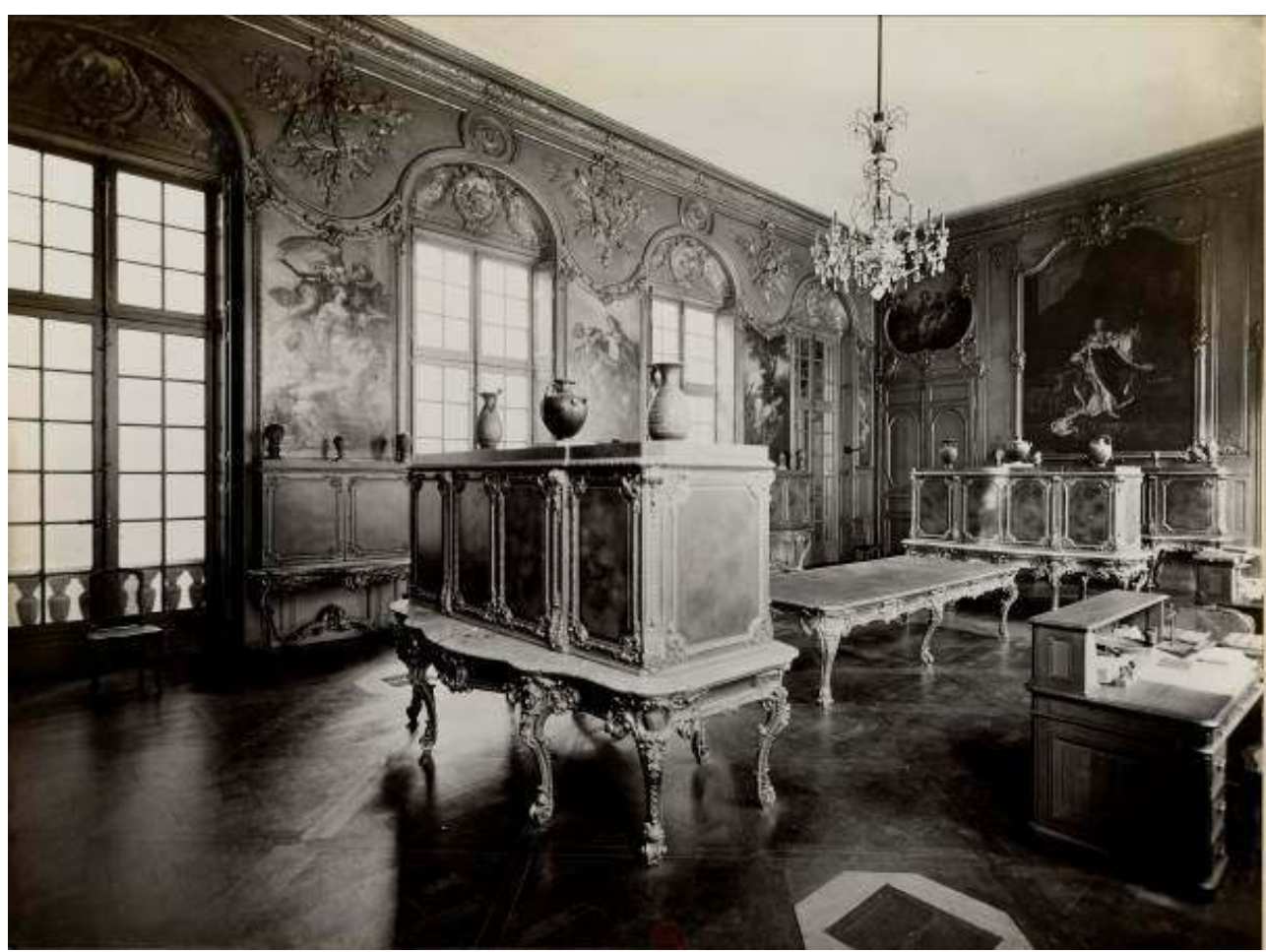

Jean-Louis Pascal. Après 1907

B.n.F., département des estampes, Va 237 (8) Fol H 23572-0028

Dans le cabinet du Roi que Jean-Louis Pascal restitue au premier étage de son nouveau bâtiment sur la rue Vivienne, au nord du pavillon central, l'architecte installe les trois trumeaux de Charles Natoire sur la rue Vivienne, entre les grandes fenêtres. Sur le côté opposé, à l'ouest, il place ceux de Carl Van Loo entre les portes garnies de miroirs qui font face aux croisées. Pascal soustrait ces trumeaux à la salle de lecture des manuscrits dont ils décoraient les entrecroisées depuis 1886. Sur le mur sud du cabinet du Roi, Jean-Louis Pascal place le Louis XIV d'après Hyacinthe Rigaud, entre les portes qui donnent dans la salle Barthélémy, portes surmontées des muses Clio et Melpomène. Au nord, entre les portes donnant dans la petite bibliothèque spéciale, il installe le Louis XV de Michel Van Loo. Ces portes sont surmontées par les muses Uranie et Erato que l'architecte a transférées, avec leurs consœurs, du vestibule d'honneur de l'administration :

«Je dois préparer une double opération [explique Pascal] dont j'ai déjà parlé dans un rapport récent: reprendre toutes les toiles de Van Loo, de Natoire, de Boucher, de Rigault, etc, dispersées précédemment dans un but de conservation et les réunir dans leur disposition primitive, leurs cadres, décors très riches qui les accompagnaient et dont tous les motifs ont été jadis complétés, restaurés, adaptés par M. Germain dans les diverses salles du fond de la cour d'honneur. Il restera des vides qui seront à remplir par ceux qui les avaient ajustés et ordonnés sous ma direction il y a une vingtaine d'années : c'est-à-dire le décorateur M. Germain et après lui le menuisier d'entretien. $»^{74}$ 
Pascal impose encore une fois Gustave Germain : «Personne n'était plus préparé que lui à mettre en œuvre les documents fournis pour aboutir à une reconstitution archéologique et artistique qui sera très appréciée, j'en suis sûre ${ }^{75}$. Pascal discute avec Léopold Delisle et Henry Marcel du thème des peintures qui vont remplacer dans le vestibule de l'administration les dessus-de-porte de François Boucher :

«Il conviendrait que les peintures, sans prétendre à ces pastiches toujours regrettables, puissent aussi s'associer à l'entourage de l'époque de la Régence que $\mathrm{j}$ 'ai pris pour thème. $\mathrm{M}$. Delisle y voyait des sujets allégoriques comme les sujets des Boucher : histoire, philosophie, etc. Il m'a suggéré des vues des différents locaux occupés par la Bibliothèque nationale depuis Charles V... Blois, Fontainebleau, etc., ce qui réclamait une recherche archéologique hors de mes compétences. » ${ }^{76}$

Pascal propose des portraits de personnages ayant honoré, dirigé, décoré, construit la Bibliothèque. L'administrateur général lui répond:

"J'imagine que des allégories pastichées de Boucher et de Natoire feraient assez pauvre dans les trumeaux du vestibule. (C'est un genre dont vos artistes ont perdu le secret et qu'ils traitent lourdement et sans grâce) [...] Des paysages, dans ces cadres chantournés, [...] seraient condamnés à des proportions plus qu'exiguës; je crains en outre avec vous, que les erreurs documentaires n'y abondent. Des portraits, alors? Mais tous les personnages portraiturables sont déjà représentés ici par des bustes. Il me semble que des attributs et des fleurs seraient les motifs les plus appropriés, par leur plasticité, aux espaces à utiliser, et que leurs colorations tendres égaieraient assez agréablement les boiseries de chêne. $»^{77}$

32 Les dessus-de-porte de François Boucher seront remplacés dans le vestibule de l'administration par des panneaux en camaïeu, dans des tons de sanguine, peints par Lucien Mignon (1865-1944) ${ }^{78}$. Ce sont les rentoileurs des musées nationaux, Brisson frères, qui nettoient et restaurent les toiles destinées au cabinet des médailles ${ }^{79}$. Dans leur bordure primitive, ces dessus-de-porte étaient encadrées d'un bandeau chantourné, décoré de volutes et de coquilles, le tout ayant à peu près la forme d'un cœur, et surmonté d'un masque à cheveux ébouriffés. Ces cadres subsistaient encore à la fin du XIX siècle $^{80}$. Les visages féminins des bordures à la chevelure se prolongeant en guirlandes de fleurs sont toujours « pleins d'assurance paisible, majestueux et rêveurs " ${ }^{81}$.

Quatre panneaux ${ }^{82}$ situés dans les écoinçons du nouveau cabinet du Roi, destinés à masquer la très légère différence de longueur de celui-ci avec le cabinet de l'arcade Colbert, ont été peints par Hector d'Espouy (1854-1928) ${ }^{83}$, peintre décorateur, professeur à l'École nationale supérieure des beaux-arts. Ils représentent des figures d'enfants se détachant sur un fond de feuillages ${ }^{84}$. Toute la serrurerie a été réalisée d'après des documents anciens :

« Les huit fenêtres et les quatre portes de la salle dans laquelle je reproduis l'ancien cabinet des médailles - avec les peintures et le mobilier d'autrefois - doivent être garnies d'espagnolettes [...] dont les modèles et motifs ont été étudiés et réalisés dans [...] le vestibule et les bureaux de M. l'administrateur. Le décorateur M. Germain y a participé autrefois; mais les ressources de M. Bricard en documents archéologiques, artistiques, etc., nous ont été d'un grand secours pour mettre en harmonie les ferrures de nos baies avec la richesse décorative des riches arrangements de l'époque de Louis XV $»^{85}$.

Les lambris sont verts et la décoration sculptée rehaussée d'or ${ }^{86}$. Dans les trumeaux et entre les portes sont placées de magnifiques tables avec plateau en marbre veiné ou consoles sur pieds chantournés supportant de grands médailliers ou armoires, sculptés et dorés. Au milieu de la salle, une grande table est attribuée à Robert de Cotte. Deux autres 
grandes tables identiques, avec médailliers, ont été exécutées à la demande d'Ernest Recoura, architecte de la Bibliothèque de 1912 à 1932. Pascal demande au menuisier Fender de réaliser un plancher en sapin en-dessous du niveau du futur plancher à feuilles qui devra être réalisé d'après celui qui existe à l'agence de la rue Colbert « avant que nous fassions la décoration même de cette salle, décoration qui va régler aussi un ensemble de fenêtres, comportant des tricheries à cause de la hauteur moindre de la couverture intérieure $»^{87}$. Pour restaurer le mobilier de l'ancien cabinet des médailles et créer de nouveaux meubles, Pascal s'adresse à l'ébéniste André Chevrie. Ce dernier restaure la grande table Louis $\mathrm{XV}$, dont les tiroirs sont à jour, en consolidant ces derniers à l'aide de parements de bois.

En 1907, Pascal n'a encore réalisé que la décoration du cabinet du Roi. Les deux autres pièces, faisant suite à cette salle, ne présentent que des surfaces brutes ${ }^{88}$. Elles ne sont toujours pas achevées en 1913, car un état des lieux réalisé par Ernest Recoura indique qu'il reste à faire ${ }^{89}$ l'aménagement intérieur de la salle centrale du cabinet des médailles au-dessus du vestibule (salle Barthélémy) avec fourniture et pose de casiers à médailles, vitrines, parquet, et bouches de chaleur. Puis dans les deux pièces à la suite, sur le jardin, l'architecte indique qu'il faut réaliser des travaux d'aménagement intérieur, garnir de chambranles, fermetures et de peintures ${ }^{90}$.

Ce n'est qu'en 1917 que les médailles prendront possession de ces nouveaux locaux qui serviront en attendant de lieu d'expositions temporaires : en 1904 se tient l'exposition des Primitifs français, la première exposition payante, qui accueille cent cinquante mille visiteurs. L'inauguration de l'exposition se déroule le mardi 12 avril 1904 au pavillon de Marsan du musée des arts décoratifs, pour les peintures, les tapis et les émaux, à la Bibliothèque nationale pour les manuscrits à miniatures exposés dans des vitrines prêtées par la société des Arts décoratifs ${ }^{91}$. À cet effet Pascal demande au menuisier Favarou la location pour six mois d'un escalier provisoire devant desservir la grande salle du $1^{\mathrm{er}}$ étage du grand bâtiment neuf sur la rue Vivienne ${ }^{92}$. Ce qui ne manqua pas de lui attirer des reproches: «Comment la Bibliothèque nationale ne peut offrir qu'une échelle de meunier dangereuse et étriquée aux étrangers qu'elle appelle à contempler une admirable collection! [...] N'auriez-vous pas pu trouver une autre salle dans votre palais des merveilles et surtout un autre escalier ? [...] Les dangers d'incendie ${ }^{93}$ sont grands avec l'escalier en bois et les parois huilées $»^{94}$. En mai 1906, une exposition sur les raretés et pièces exceptionnelles du XVIII ${ }^{e}$ siècle, fut réalisée avec le concours de prêteurs du dehors, autre innovation.

L'arrêté du 2 mars 1925, inscrivit à l'Inventaire supplémentaire des monuments historiques la décoration intérieure du nouveau cabinet des médailles ${ }^{95}$.

\section{NOTES}

1. Léon Labrouste, «Bibliothèque nationale rue de Richelieu, à Paris », R.G.A.T.P., $\mathrm{n}^{\circ} \mathrm{XXXV}$, 1878, note 2 p. 149. Des sondages effectués en juillet 2008 dans l'ex-rotonde du département des médailles ont permis de le vérifier: on a retrouvé, sous la toile 
marouflée, des motifs décoratifs identifiés par Marc Le Cœur comme étant ceux d'une planche dessinée d'Henri Labrouste conservée au département des Estampes. Voir Marc Le Cœur, «Histoire d'un palimpseste », Revue de la Bibliothèque nationale de France, $\mathrm{n}^{\circ} 30$, 2008, 96 p., p. 31.

2. Parlant de Pascal, Henri Bouchot dit : «Il faut un grand courage, et, je le disais, un tempérament énorme pour faire ainsi abstraction de soi, se condamner à un "ressemelage", quand on a en soi des œuvres prêtes à sortir et qui attendent. C'est le cas de M. Pascal, successeur de Labrouste, ce sera le plus grand honneur de sa carrière; au lieu de méditer la transformation, de projeter l'anéantissement des choses d'autrefois, de suivre la route qu'on lui avait indiquée, M. Pascal a fait œuvre de bénédictin, a reconstitué sa chronique, s'est piqué d'érudition, et, fort capable de mieux, sans aucun doute, s'est voué à redire les phrases d'autrui, mot à mot, sans rien y changer, en ne créant que l'indispensable et dans le style adopté par les vieux ». Henri Bouchot, «Les derniers travaux de décoration exécutés à la Bibliothèque nationale par $\mathrm{M}$. Pascal, architecte ", Revue des arts décoratifs, $12^{\mathrm{e}}$ année, 1891-1892, p. 81-97, p. 95.

3. Le devis fait état de : « Exécution en pierre : 3 linteaux des fenêtres sur la rue Vivienne, 6 grandes consoles, 3 crosses clés à piastres, 1 fronton couronne et palme, 2 rosaces des crossettes de la table sur l'angle, 10 feuilles palmettes du chéneau sur la rue Vivienne ». Arch. adm. B.n.F., 2011/001/10009, approbation d'une soumission de sculpture décorative, 21 janvier 1901, lettre du ministre de l'instruction publique et des beaux-arts à Pascal. En 1905, les travaux de sculpture décorative sur la rue Vivienne sont évalués à $23650 \mathrm{fr}$.

4. Arch. adm. B.n.F., 2011/001/10011, lettre de Pascal au sous-secrétaire d'État aux beauxarts, 26 mai 1905. Le sculpteur Louis Lavy avait la protection du ministère et fut imposé à Pascal.

5. Encore appelé salle Barthélémy, il succède au cabinet du Roi dans l'aile Vivienne, au sud.

6. «La médaille de Louis XIV est terminée et moulée avec les retouches que vous désiriez. Le Lionel d'Este a sa chevelure très modifiée. Le metteur au point est prêt. Vous pouvez donc faire poser les échafaudages et quand ils seront prêts, pour ne pas perdre de temps, faire avertir M. Guigues, 10 passage de la Gaité, qui mettra le travail en route. » Arch. adm. B.n.F., 2011/001/10021, lettre de Bottée à Pascal, 2 juillet 1906.

7. Arch. nat., $\mathrm{F}^{21} 5863$, lettre du sous-secrétaire d'État aux beaux-arts : Dujardin-Beaumetz à Pascal, 13 janvier 1906.

8. « Il ne s'agit pas d'un simple grandissement au diamètre de $80 \mathrm{~cm}$ que doivent avoir ces sculptures; l'amplification et l'association avec le décor général demandent l'adaptation par une main experte, c'est-à-dire pour chacune des médailles un modèle et une exécution sur place absolument parfaite. Je crois que le prix pourrait être de deux mille fr. par type, soit 10000 fr. pour les cinq ». Arch. adm. B.n.F., 2011/001/10075, lettre de Pascal au sous-secrétaire d'État aux beaux-arts, 29 décembre 1905. Louis Bottée habite 16 rue fontaine Saint-Georges, Paris.

9. Arch. nat., $\mathrm{F}^{21} 5863$, commande de cinq médaillons pour la décoration de la façade sur jardin, lettre de Pascal au sous-secrétaire aux beaux-arts, 29 décembre 1905.

10. Il en a forgé le terme dans Le Magasin pittoresque de décembre 1852 à propos de l'œuvre de Ledoux. Robin Middleton, David Watkin, Architecture du XIX ${ }^{e}$ siècle, Paris-Milan, Gallimard/Electa, 1993, 403 p., p. 233-234. 
11. Ernest Babelon fut conservateur en chef au cabinet des médailles de 1892 à 1924 .

12. Arch. adm. B.n.F., 2011/001/10021, lettre de Pascal au sous-secrétaire d'État aux beaux-arts, 15 janvier 1906.

13. «M. Bottée a terminé à ma satisfaction les cinq médailles qui signaleront le cabinet des médailles à l'extérieur, lorsqu'on aura pu l'installer au premier étage du bâtiment de la Bibliothèque nationale sur la rue Vivienne ». Arch. adm. B.n.F., 2011/001/10022, lettre de Pascal au sous-secrétaire d'État aux beaux-arts, 2 février 1907.

14. «Les études des deux consoles de la porte d'entrée étant terminées, on commence à mouler mardi. [...] J'ai besoin d'autre part qu'on remonte promptement l'échafaudage dans la première travée entre les colonnes, à droite, en regardant la façade, de façon à pouvoir exécuter, dans le gros bossage laissé à la clef, la médaille de la République française; et que le tout soit fini vers le 8 du mois prochain avant l'ouverture de l'exposition qui va avoir lieu dans les salles du $1^{\text {er }}$ étage. [...] Comme les autres modèles ne sont pas prêts, ce n'est qu'après la fermeture de l'exposition, fin octobre, que nous combinerons pour l'ensemble des autres fenêtres un échafaudage en bascule s'appuyant sur le bandeau, peut-être sur l'imposte des fenêtres, lorsque nous aurons repris possession de la salle pour l'achever. » Arch. adm. B.n.F., 2011/001/10021, lettre de Pascal à Auget, 11 avril 1906.

15. Les dimensions sont : 4,30 m. $\times 2,48 \mathrm{~m}$.

16. Les dimensions sont: $6,35 \mathrm{~m}$. x 3,25 m. Le prix est de 2 fr. le kilo pour la partie métallique et de $10 \mathrm{fr}$. le kilo pour les bronzes d'encadrement et battements extérieurs. Arch. adm. B.n.F., 2011/001/10011, lettre de Baudet et Donon à Pascal, 22 novembre 1905.

17. T. Parizeaux, 44 rue Saint-Sabin, Paris.

18. Arch. nat., $\mathrm{F}^{21} 5863$, commande de cinq médaillons pour la décoration de la façade sur jardin, lettre de Pascal au sous-secrétaire aux beaux-arts, 30 mai 1906. La maison Lelièvre est située au n ${ }^{\circ} 13$ de la rue des Petits-Champs.

Les lettres ont pour dimensions : 0,22 m. x 0,18 m x 0,14 m. (épaisseur). Arch. adm. B.n.F., 2011/001/10097, devis du 25 mai 1906.

19. Fourniture de 3 linteaux : 3,70 x 0,75 x 1,00 et 2 linteaux : 3,50 x 0,75 x 1,00 en pierre de Tercé par Fèvre et $C^{\text {ie }}, 237$ rue Lafayette, Paris, Arch. adm. B.n.F., 2011/001/10010, lettre de Fèvre à Auget, 23 avril 1903.

20. Les colonnes $(4,70 \times 0,65)$ valent 2100 à 2500 fr. pièce et les pilastres $(4,70 \times 0,65 \mathrm{x}$ 0,12 ) valent 625 fr. pièce, en 2 ou 3 morceaux, car il est impossible de les avoir monolithes. Arch. adm. B.n.F., 2011/001/10011, lettre de G. Leclercq à Pascal, 23 janvier 1904.

21. Ce sont les deux premières salles de l'aile Vivienne, au nord.

22. Arch. adm. B.n.F., 2011/001/10023, lettre de Pascal à Ernest Babelon, 25 décembre 1908.

23. C'est ainsi qu'elle apparaît sur le plan de la Bibliothèque nationale en 1850 avant les transformations de Labrouste.

24. Arch. adm. B.n.F., 2011/001/10021, lettre de Pascal au sous-secrétaire d'État aux beaux-arts, 26 mars 1906.

25. Ibid., lettre de Pascal au sous-secrétaire d'État aux beaux-arts, 25 mars 1906.

26. Arch. adm. B.n.F., 2011/001/10023, lettre de Pascal à Henri Deglane, 8 décembre 1908. 
27. Arch. nat., $\mathrm{F}^{21} 5856$, lettre de J.-L. Pascal au secrétaire d'État aux beaux-arts, 12 juin 1910.

28. Ibid., lettre de Pascal au secrétaire d'État aux beaux-arts, 18 septembre 1911.

29. Arch. adm. B.n.F., 2011/001/10097, lettre de L. Bonfils à Pascal, 4 décembre 1911, fourniture de cinq lucarnes en zinc, et devis de Germain fils.

30. Arch. adm. B.n.F., 2011/001/10026, arrêté de mise en demeure de l'entreprise Auget, du ministre de l'instruction publique et des beaux-arts, 26 avril 1911.

31. Son diamètre est de 3,20 m. Pascal avait hésité à encercler la base de la coupole d'un chaînage métallique.

32. À la mort de Gustave Germain, en 1909, son troisième fils reprend l'atelier et fonde une société de sculpteurs avec Alfred Chatillon de 1910 à 1919. Voir Gustave Germain, sculpteur ornemaniste (1843-1909), biographie de Michel-Gasse à paraitre.

33. Arch. adm. B.n.F., 2011/001/10076, lettre de Recoura au sous-secrétaire d'État, 13 septembre 1912. La pierre de Lavoux est proche de la pierre de Tercé.

34. «Les livres de François $1^{\text {er }}$ expliquent la présence du roi, la galerie Mazarine justifie Mazarin [sans doute a-t-on changé par la suite pour Richelieu, la rue principale passant devant la B.N. portant son nom], les constructions de Robert de Cotte [ont été réalisées sous] Louis XV, dont on a de charmantes effigies». Arch. nat., $\mathrm{F}^{21} 5863$, lettre de J.-L. Pascal au sous-secrétaire d'État aux beaux-arts : Dujardin-Beaumetz, 25 septembre 1911. Le sculpteur est Louis Bottée.

35. Né en 1865 en Suisse, élève de Pascal à l'E.B.A. (promotion 1885), il est diplômé en 1892.

36. La maison Vaugirard, fondée en 1868, a marqué le paysage de Paris avec la création des premières briques flammées, reconnaissables au $\mathrm{V}$ frappé sur leur chant. La teinte la plus connue est : Terre de Rose.

37. Arch. adm. B.n.F., 2011/001/10025, lettre d'Auget à Emery, 7 avril 1910.

38. Arch. adm. B.n.F., 2011/001/10075, lettre de Pascal au contrôleur général, 31 juin 1903.

39. Arch. adm. B.n.F., 2011/001/10340, lettre du 9 décembre 1903 de Favarou à Pascal.

40. Le cabinet du Roi est situé dans l'aile Vivienne après la petite bibliothèque spéciale, $3^{\mathrm{e}}$ pièce au nord.

41. Arch. adm. B.n.F., 2011/001/10340, lettre de Favarou à Pascal, 25 janvier 1906.

42. Arch. adm. B.n.F., 2011/001/10023, petite note.

43. Ibid., lettre de Pascal au sous-secrétaire d'État, 23 mai 1908.

44. Ibid., lettre du sous-secrétaire d'État à Pascal, 26 mai 1908.

45. Ibid., lettre du vérificateur Didelot à Pascal, 19 juin 1908.

46. Ibid., petite note.

47. Arch. adm. B.n.F., 2011/001/10025, lettre de Pascal à Auget, 8 novembre 1910.

48. Ibid., lettre de Pascal au sous-secrétaire d'État aux beaux-arts, 15 novembre 1910.

49. Ceci est corroboré par la date de » $1870 »$ inscrite sur le palier du premier étage, en haut de l'escalier d'honneur, sur le mur qui fait face au départ de la galerie reliant ce bâtiment du fond de la cour d'honneur et celui longeant la rue de Richelieu, ancienne "galerie de bois ». 
50. Ce que l'on appelait la "baignoire», béance de forme oblongue qui permettait d'éclairer le rez-de-chaussée de l'administration. Elle fut bouchée en 1926 par Ernest Recoura au moyen de carreaux de verre quand il réaménage la galerie Mazarine et son palier grâce au legs Blumenthal. Le garde-corps qui l'entourait fut aussi détruit.

51. Léon Labrouste, La Bibliothèque nationale, son début et ses accroissements, ses bâtiments et ses constructions, ses agrandissements, ses travaux, Paris, H. Luthier, 1885, 94 p., p. 71-72.

52. Arch. adm. B.n.F., 2011/001/10076, lettre de Pascal au sous-secrétaire d'État aux beaux-arts, 6 mars 1911.

53. Le $1^{\text {er }}$ mars 1910, Ernest Hébrard (1875-1933), (ancien grand prix de Rome), auditeur au conseil général des bâtiments civils, est nommé architecte ordinaire à l'agence des travaux d'agrandissement de la Bibliothèque nationale, en remplacement d'Ernest Herscher mis en congé sur sa demande.

54. Arch. adm. B.n.F., 2011/001/10026, lettre d'Ernest Hébrard à Pascal, 11 août 1911.

55. Arch. adm. B.n.F., 2011/001/10076, lettre de Pascal au sous-secrétaire d'État aux beaux-arts, 29 avril 1912.

56. Ibid., lettre de Recoura à Pascal, 7 mai 1912.

57. Ibid., lettre de Recoura au sous-secrétaire d'État aux beaux-arts, 3 février 1913.

58. Arch. adm. B.n.F., 2011/001/10021, lettre de Pascal à L. Lambeaux, secrétaire de la Commission du vieux Paris, 15 janvier 1906.

59. Arch. adm. B.n.F., 2011/001/10021, lettre de Recoura au sous-secrétaire d'État aux beaux-arts, 8 octobre 1913.

60. Arch. adm. B.n.F., 2011/001/10010, lettre de Pascal, 26 juin 1903.

61. Un projet de cession d'une partie du dépôt des marbres par le ministre des beaux-arts à celui des finances, en vue de l'installation d'un dépôt et d'une salle de vente des Domaines, obligea au transfert des globes. Henri Deglane proposa alors à Pascal de les envoyer à Versailles : « est-ce que Versailles qu'on transforme de plus en plus en musée ne se trouverait pas indiqué ?»; Arch. adm. B.n.F., 2011/001/10023, lettre de Deglane à Pascal, 7 septembre 1908. La salle laissée libre sera réservée à des expositions publiques des départements des médailles et estampes.

62. Arch. nat., $\mathrm{F}^{21} 5854$, lettre de Recoura au secrétaire d'État aux beaux-arts, 7 décembre 1915.

63. Elle mesure 18, $30 \mathrm{~m}$. sur 12, $35 \mathrm{~m}$. Elle était haute à l'origine de 7, $45 \mathrm{~m}$.

64. Ses proportions sont de : $11,30 \mathrm{~m}$. par $8,90 \mathrm{~m}$.

65. Degeorge a effectivement sculpté une Médaille frappée à la mémoire des élèves de l'École des beaux-arts tués pendant la guerre de 1870-1871. Une épreuve de cette médaille a été donnée au musée du Luxembourg par Oscar Roty. Stanislas Lami, Dictionnaire des sculpteurs de l'École Française au XIX ${ }^{e}$ siècle, Paris, éd. Honoré Champion, 1916, 4 vol. , vol. 2, p. 140.

66. Lettre de Jean-Louis Pascal à G. Germain, 12 octobre 1908, appartenant aux descendants de G. Germain, Patrice et Alexandra. Elle m'a été aimablement prêtée par Michel-Gasse, son biographe. Je les en remercie vivement tous trois.

67. La donation se composait de : 6893 médailles, 373 camées, pierres gravées et cylindres, 189 bijoux en or, 39 statuettes de bronze grecques, étrusques et romaines, 43 armures et armes antiques, 85 vases étrusques et grecs, des marbres dont un torse de Vénus, une tête 
de statue romaine en bronze. « Don du duc de Luynes », Le Moniteur Universel, 3 décembre 1862, B.n.F., dp. Mss, N.a.fr., 13026, f. 38.

68. La salle de Luynes mesure : $11,25 \mathrm{~m} . \times 8,90 \mathrm{~m}$.

69. Elle mesure : 20, $53 \mathrm{~m} . \times 8,90 \mathrm{~m}$.

70. En 1900, l'ébéniste Chevrie livre « un grand meuble à médailles en acajou verni et ornements en cuivre, comprenant un grand piètement à neuf pieds, et le corps du haut, renfermant douze tiroirs avec boutons et porte-étiquettes au prix soumissionné de 7000 francs ». Arch. nat., $\mathrm{F}^{21}$ 2934, direction des beaux-arts, travaux exécutés à la B.N., M. Pascal architecte, bordereau des mémoires, 1900.

71. Arch. adm. B.n.F., 2011/001/10076, adjudication du 26 mai 1913, lettre de Recoura au sous-secrétaire d'État aux beaux-arts, 8 octobre 1913.

72. Lambris et mobilier furent réalisés par Verbeckt et ses employés de 1741 à 1742. Thierry Sarmant, Le Cabinet des médailles de la Bibliothèque nationale, 1661-1848, Paris, École des chartes, 1994, 403 p., p. 168.

73. Ibid., p. 169-170.

74. Arch. adm. B.n.F., 2011/001/10009, lettre du 26 novembre 1901 de Pascal au ministre. Le devis s'élève à $8000 \mathrm{fr}$.

75. Arch. adm. B.n.F., 2011/001/10010, lettre du 4 avril 1902 de Pascal au ministre.

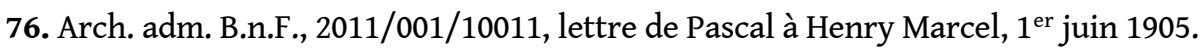

77. Ibid., lettre de Henry Marcel à Pascal, 2 juin 1905.

78. Arch. nat., $\mathrm{F}^{21} 5863$, dossier: "Bibliothèque nationale. Commande de quatre médaillons en camaïeu ». La dorure des quatre cadres du vestibule de l'administration est exécutée par Herreman pour 700 fr. Arch. adm. B.n.F., 2011/001/10011, lettre de Herreman à Pascal, 4 sept 1905.

79. Le devis fait état de: 4 panneaux contournés de Boucher de 1,80 m. x 1,80 m.; 6 panneaux en hauteur de Van Loo et Natoire de 2,50 m. x 1,40 m.; 2 grands panneaux de Louis XIV et Louis XV. Arch. adm. B.n.F., 2011/001/10011, devis de Brisson frères, rentoileurs des musées nationaux, 20 quai de la Mégisserie et 2-4 rue de la Bourdonnais, 26 janvier 1904.

80. Henri Bouchot, «Les derniers travaux de décoration exécutés à la Bibliothèque nationale par M. Pascal, architecte », op. cit., p. 93.

81. Thierry Sarmant, Le Cabinet des médailles de la Bibliothèque nationale, 1661-1848, op. cit., p. 173.

82. Ils mesurent $0,55 \mathrm{~m}$. de large sur $2,6 \mathrm{~m}$. de haut.

83. Hector d'Espouy, 1 rue de Fleurus, Paris ( $\left.6^{6}\right)$.

84. Arch. nat., $\mathrm{F}^{21} 5863$, lettre de Recoura au ministre de l'instruction publique et des beaux-arts, 13 juillet 1918 .

85. Arch. adm. B.n.F., 2011/001/10075, lettre de Pascal au sous-secrétaire d'État, 6 décembre 1901.

86. La réfection des peintures du cabinet du Roi a été réalisée grâce au don de $15500 \mathrm{fr}$. de M. et Mme Charles Prince à la » Société des Amis » de la Bibliothèque. Arch. nat., $\mathrm{F}^{21}$ 5862, lettre de l'administrateur général Roland-Marcel à Paul Léon, directeur des beauxarts, 15 octobre 1927.

87. Arch. adm. B.n.F., 2011/001/10009, lettre de Pascal à Fender, 22 septembre 1901. 
88. Henry Marcel, Henri Bouchot, Ernest Babelon, Paul Marchal, Camille Couderc, La Bibliothèque nationale, Paris, éd. H. Laurens, 1907, 129 p., p. 15.

89. À l'aide d'un crédit de 400000 francs inscrit au chapitre 86 du budget de l'exercice 1913.

90. Arch. adm. B.n.F., 2011/001/10076, lettre de Recoura au sous-secrétaire d'État aux beaux-arts, 3 février 1913.

91. Arch. adm. B.n.F., 2011/001/10340, lettre de Pascal à Metman, conservateur-secrétaire de la société des Arts décoratifs, 12 janvier 1904.

92. Pour 2300 francs. Ibid., lettre du 9 décembre 1903 de Favarou à Pascal.

93. L'incendie de la Bibliothèque nationale de Turin le 25 janvier 1904 avait frappé les esprits:»Que dites-vous du sinistre de Turin? Voilà qui n'est guère engageant pour l'introduction de l'électricité dans les bibliothèques ", ibid., lettre de Pascal à Mortreuil, 29 janvier 1904.

94. Ibid., lettre d'un conseiller général de Paris au directeur de la Bibliothèque nationale, 12 avril 1904.

95. Arch. nat., $\mathrm{F}^{21} 5863$, note du chef du bureau des monuments historiques pour le chef du bureau des bâtiments civils, Palais-Royal, 13 mars 1925.

\section{RÉSUMÉS}

Jean-Louis Pascal achève le "carré Vivienne " dans les dix premières années du XX $\mathrm{XX}^{\mathrm{e}}$ siècle. Il adopte sur la rue Vivienne la même ordonnance que sur la deuxième partie de la rue Colbert, baies simples et riches surmontant un rez-de-chaussée à refends. Les clés des baies du salon central sont ornées de médailles annonçant la destination de l'édifice, tout comme sur l'aile donnant sur le jardin. Un nouvel escalier dessert désormais le cabinet des médailles. Quatre premières salles, la salle des colonnes, appelée tout d'abord «balcon des globes Coronelli », la salle du Grand Camée dont Pascal orne les angles du plafond de grosses médailles, la salle de Luynes et la salle Barthélémy sont créées par Pascal pour conserver médailles et antiques. Dans la dernière salle, Pascal, poursuivant son œuvre de « bénédictin » restitue le cabinet du Roi tel qu'il se trouvait dans l'arcade Colbert: il y place les trumeaux de Charles Natoire et Carle Van Loo et les muses de François Boucher. Louis XIV et Louis XV se font de nouveau face et les boiseries sculptées de l'ancien cabinet ornent à nouveau ce cabinet royal qui sera inscrit dès 1925 à l'Inventaire supplémentaire des monuments historiques.

Jean-Louis Pascal completes his work at the "Carré Vivienne » in the first ten years of the $\mathrm{XX}^{\text {th }}$ century. He develops the same architectural organization on the Rue Vivienne as he did for the second part of the Rue Colbert, riche and simple bays on a ground floor with a ridge. Sides of the bays are adorned with medallions related to the building's use, as is the wing on the garden. A new staircase leads to the Cabinet des médailles. For keeping the cameos and antic artefacts, Pascal establishes the following first four rooms : the Colonn Room, firstly called "The Coronelli's globs room", the "Great Cameo's room" adorned with huge medallions on the ceiling corners, the "Luynes room" and the "Barthélémy room". In the last room, Pascal, following his "benedictus" 
target, restitutes the "Cabinet du roi" as he used to be in the Rue Colbert : the Charles Natoire's and Carle Van Loo upright posts, and the François Boucher's muses. Louis the XIV ${ }^{\text {th }}$ and Louis the $\mathrm{XV}^{\text {th }}$ are facing each other, and sculpted woodworks of the former Cabinet des médailles find their place again in the royal Cabinet, which was going to be protected by the cultural heritage legislation in 1925.

Jean-Louis Pascal vollendet das "Carré Vivienne" im ersten Jahrzehnt des 20. Jahrhunderts. Er verwendet für die Fassade zur Rue Vivienne die gleiche Säulenordnung wie beim zweiten Teil der Rue Colbert, bestehend aus alternierenden einfachen und verzierten Arkaden, die sich über den Zwischenwänden des Erdgeschosses erheben. Die Schlusssteine des zentralen Salons sind mit Medaillons verziert, die sich auf die Bestimmung des Hauses beziehen, ebenso im Gartenflügel. Eine neu errichtete Treppe führt nunmehr zum Münzkabinett. Pascal entwirft vier Säle für die Münz- und Antikensammlungen, einen ersten Saal, den Säulensaal, der zunächst nach den Coronelli-Globen benannt wird, dann den Grand-Camée-Saal, dessen Deckenwinkel von Pascal mit großen Medaillons versehen werden, den Luynes-Saal sowie den Barthélémy-Saal. In einem letzten Saal stellt Pascal in geradezu als "benediktinisch" zu bezeichnender Weise das Kabinett des Königs in der Form wieder her, in welcher es sich bereits in den Colbert-Arkaden befand: Er bringt darin die Trumeau-Pfeiler von Charles Natoire und Carle Van Loo unter sowie die Musen von François Boucher. Damit vereint Pascal quasi aufs Neue Ludwig XIV. und Ludwig XV. in einem Raum und erweckt die skulptierte Holztäfelung des alten Cabinet zu neuem Leben, im Rahmen dieses neu erschaffenen Cabinet royal, das 1925 in das Inventaire supplémentaire des Denkmälerverzeichnisses aufgenommen wird.

\section{INDEX}

Index chronologique : époque contemporaine, XXe siècle

Mots-clés : restitution

Keywords : restitution

Schlüsselwörter : Wiederherstellung

\section{AUTEUR}

\section{ANNE RICHARD-BAZIRE}

Anne Richard-Bazire est docteur de l'École pratique des hautes études (section des sciences historiques et philosophiques), diplômée en histoire de l'art contemporain et en archéologie égyptienne. Elle est actuellement chargée du cours de synthèse de l'Histoire de l'architecture occidentale, $\mathrm{XIX}^{\mathrm{e}}-\mathrm{XX}^{\mathrm{e}}$ siècles, à l'École du Louvre. Elle a rédigé différents articles : « L'habitat néo-gothique à Paris au XIX ${ }^{\mathrm{e}}$ siècle ", La demeure médiévale à Paris, Étienne Hamon et Valentine Weiss (dir.), Paris, 2012, Archives Nationales, p. 250-257 ; « La salle Ovale ou l'histoire des dysfonctionnements dans les chantiers parisiens du XIX ${ }^{\mathrm{e}}$ siècle à travers l'exemple de la Bibliothèque nationale ", La Collégialité et les dysfonctionnements dans la décision administrative, JeanMichel Leniaud et François Monnier (dir.), Paris, 2011, École Pratique des Hautes Études, p. 103-112; " Jean-Louis Pascal ou le respect du patrimoine ", article mis en ligne sur le site "Archicab", le 4 décembre 2011, http://www.jeanfrancoiscabestan.com/bnfrichelieu.html ; « Le concours pour la reconstruction de l'Hôtel de Ville de Paris (1872-1873), un échec pour l'architecte Jean-Louis Pascal », Livraisons d'histoire de l'architecture, $\mathrm{n}^{\circ}$ 19, 2010, p.111-134 ; « L'escalier d'honneur de la Bibliothèque nationale de la rue de Richelieu ", Sites et Monuments, $\mathrm{n}^{\circ}$ 
207, oct.-nov.-déc. 2009, p. 22-24 ; « La salle Ovale », Revue de la Bibliothèque nationale de France, $\mathrm{n}^{\circ}$ 30, 2008, 96 p., p. 32-39 ; «La faculté de médecine et de pharmacie de Bordeaux par Jean-Louis Pascal (1876-1888 et 1902-1922)», Livraisons d'histoire de l'architecture, $\mathrm{n}^{\circ} 13,2007, \mathrm{p} .105-120$; « Le ministère de l'agriculture de la rue de Varenne par Emmanuel Brune, l'alliance de la science et de l'art ", Livraisons d'histoire de l'architecture, $\mathrm{n}^{\circ}$ 8, 2004, p. 81-94 ; « Le parvis de Notre-Dame » et « Le square Jean XXIII », Autour de Notre-Dame, sous la dir. de Alain Erlande-Brandeburg, Jean-Michel Leniaud, François, Christian Michel, Paris, D.A.A.V.P., 2003, 269 p., p. 240-242 et 243-245 ; « Les réalisations de Pascal », et « L'École des beaux-arts » avec Fabienne Doulat, Les bibliothèques parisiennes, architecture et décor, sous la dir. de Myriam Bacha et Christian Hottin, Paris, D.A.A.V.P., 2002, 272 p., p. 115-117 et 132-134; « Un siècle de réflexion sur la construction des bibliothèques ", Des palais pour les livres, Labrouste, Sainte-Geneviève et les bibliothèques, sous la dir. de Jean-Michel Leniaud, Paris, éd. Maisonneuve \& Larose, 2002, 189 p., p. 58-69 ; « Jean-Louis Pascal et la création de la salle des périodiques de la Bibliothèque nationale (1883-1936) », Livraisons d'histoire de l'architecture, $\mathrm{n}^{\circ}$ 1, 2001, p. 105-125. Elle a dirigé avec Martin Drouin : La Sélection patrimoniale, Québec, Éditions MultiMondes, coll. Cahiers de l'Institut du patrimoine de l'UQAM, n 13, 2011, 388 p. Elle a participé en octobre 2001 au colloque international organisé par la Bibliothèque Sainte-Geneviève à Paris : « Henri Labrouste et la Bibliothèque Sainte-Geneviève, Histoire et actualité », en octobre 2009 au colloque : «Victor Laloux, un architecte dans sa ville » organisé à Tours par le Conseil général d'Indre-et-Loire, en juillet 2010 aux journées d'études organisées par le Centre-Ledoux-Université Paris I, la Commission du Vieux Paris et l'Association pour la Sauvegarde et la Mise en valeur du Paris Historique, sur le thème : «BNF Richelieu : un projet en questions »; en octobre 2010, elle a été la coordinatrice scientifique avec Martin Drouin de la Sixième Rencontre internationale des jeunes chercheurs en patrimoine sur le thème de la " Sélection patrimoniale », organisée conjointement par l'Université du Québec à Montréal et l'École des Chartes à Paris, sous la direction de Luc Noppen, Lucie K. Morisset (UQAM) et JeanMichel Leniaud (École des chartes). Elle a participé en janvier 2011 aux Journées annuelles de l'équipe Histoire du droit public et de l'administration, EPHE, sous la direction de François Monnier, directeur de l'UR, Jean-Michel Leniaud et Jean-Miguel Pire sur le thème : « Les dysfonctionnements dans la décision administrative », en novembre 2011 à la journée d'études de l'équipe Histara de l'EPHE, organisée à l'INHA par Jean-François Belhoste, directeur d'études à l'EPHE, et Isabelle Parizet, maître de conférences à l'EPHE, sur le thème : « Architectures bancaires : entre le Beau et l'Utile ", en décembre 2012 au colloque international organisé par l'INHA, Le musée d'Orsay et la Maison des centraliens : «William Le Baron Jenney (1832-1907). De l'École centrale de Paris aux gratte-ciel de Chicago ». Elle a réalisé de mars 2012 à janvier 2013 pour le compte de l'OPPIC les recherches historiques préalables à la restauration des galeries Mansart, Mazarine et de la « chambre de Mazarin » à la Bibliothèque nationale Richelieu sous la direction de l'architecte en chef des monuments historiques A. de Saint-Jouan. Adresse électronique : anne.richardbazire@hotmail.com. 